This is a peer-reviewed, accepted author manuscript of the following paper: Thanh T.X. Tran and Ekin Ozer (2021), Mechanical Systems and Signal Processing. Elsevier.

\title{
Synergistic Bridge Modal Analysis Using Frequency Domain Decomposition, Observer Kalman Filter Identification, Stochastic Subspace Identification, System Realization Using Information Matrix, and Autoregressive Exogenous Model
}

\author{
Thanh T.X. Tran ${ }^{\mathrm{a}}$, Ekin Ozer ${ }^{\mathrm{b}, \mathrm{c}^{*}}$ \\ a Department of Civil Engineering, Yokohama National University, Yokohama, Japan \\ $b$ Department of Civil and Environmental Engineering, University of Strathclyde, Glasgow, UK \\ c Formerly, Department of Civil Engineering and Engineering Mechanics, Columbia University, New \\ York, US \\ *Correspondence to E-Mail: eo2327@columbia.edu; Tel.: +44 7575761119
}

\begin{abstract}
This paper presents multiple system identification of large-scale bridge structures proposing the combined usage of different modal parameter findings, namely from Frequency Domain Decomposition, Observer Kalman Filter Identification/Eigensystem Realization Algorithm, Combined Deterministic Stochastic Subspace Identification, System Realization Using Information Matrix, and Autoregressive Exogenous Model. A method-centric democratic ranking approach visualizes and quantifies the harmony among different system identification methods in terms of modal parameters, then ranks them based on the correlation among each other, and consequently complies with the highest rank modal parameter outputs. The synergistic scheme is applied on a numerical beam and two bridge structures including one healthy and another subjected to progressive damage. Looking at the top-rank selections, one can see that outlier identification results from a population of modal parameters can intuitively become extinct. The collaboration among methods is dependent on the chosen methods; therefore, method selection relies on care and fair representation of the identification features. Lack of agreement between methods can indicate low confidence in the outranking method and is quantified by median absolute deviation. Nevertheless, the majority of the algorithm population agrees on specific results, which are valuable to produce state knowledge despite low signal to noise ratio, especially without the presence of a reference. Thus, the collaborative usage of multiple methods in a systematic and ranking-based manner reduces significant error and outlier possibilities in modal identification due to algorithm-related issues, which is the novel contribution of this study.
\end{abstract}

Keywords: System Identification, Frequency Domain Decomposition, Observer Kalman Filter Identification, Combined Deterministic Stochastic Subspace Identification, System Realization Using Information Matrix, Autoregressive Exogenous Model 


\section{Introduction}

With the rise in sensor technology and computational power in the last four decades, structural health monitoring (SHM) becomes an indispensable field in civil engineering applications. Moreover, assessment and maintenance of urban infrastructure become essential, as many building stocks are subject to deteriorating effects such as natural hazards, fatigue, operational loads, and more. Besides, observing structural condition gains crucial importance, knowing that the modern structural design allows a certain extent of controlled damage for commercial and architectural purposes. In other words, engineering design targets to meet the safety criteria by keeping the structural performance above a certain level, and such qualification requires investigation using the state-of-the-art analytical and experimental capabilities.

To monitor the structural integrity of existing buildings or estimate structural safety under damaging events, a wide variety of methodologies and techniques are developed. For example, in earthquakeprone regions, seismic performance assessment is conducted to determine whether the structure meets the requirements of codes and regulations. Such a procedure heavily relies on modelling assumptions that may deviate from the reality resulting from many sources of uncertainties. Visual inspection is another exemplary application for post-event damage assessment. However, it is challenging to practice it efficiently since it requires a long and detailed inspection procedure with engineers' presence. This leads to subjectivity in results, which is unwieldy to quantify the structural conditions.

The advent of SHM brought several solutions to understand civil infrastructure's status to a better extent. For instance, compared with the models which solely depend on design drawings and guidelines, it is seen that updated models using SHM tools can provide much more accurate solutions in terms of performance assessment. What is more, considering visual inspection as a qualitative and subjective damage identification method, it is evident that SHM can offer complementarily modernized and technology-driven results in a rapid, remote, automated, and objective fashion.

As the nature of monitoring problem changes according to the application and discipline, there has been a wide variety of existing SHM approaches. (Doebling S. W., 1996) and (Carden, 2004) presented literature reviews to provide the essence, previous work, and future goals of SHM. To provide a framework for a typical SHM methodology (Rytter, A., 1993) divided SHM content into four main elements. These elements attempted to lead SHM researchers to seek answers to the following questions:

-Level 1, Detection: Is there any damage introduced to the structure?

-Level 2, Localization: If so, where is the damage concentration?

-Level 3, Quantity: To what extent is the structure damaged?

-Level 4, Consequence: What is the safety level or remaining useful life of the structure?

Following these definitions, SHM society agreed on a standard set of goals which can reduce the ongoing ambiguity in SHM literature.

In the past four decades, numerous SHM, system and modal (Peeters, 2001), (Reynders E. , 2012), as well as damage identification methodologies (Salawu, 1997), (Doebling S. W., 1998) have been introduced, yet, there is still limited access to systematically combined usage of multiple methods. ERA/OM, N4SID, and MOESP methods have been compared based on a small-scale structure (Abdelghani, 1998). Modal analysis results of a benchmark bridge are collected from different researchers pursuing a variety of methods (Peeters B., 2003). ERA/DC, least squares, and SSI based approaches are implemented on a laboratory model, and identified parameters are compared (Giraldo, 
2009). In addition to small scale and experimental studies, four different identification techniques (FDD, ERA, SSI, P-LSCF) are applied to real-life structures with comparative results (Rahman, 2012). Automation initiatives in modal identification have compared SSI-based modal analysis results with FDD (Ubertini F. G., 2013). Despite, all these efforts, an automated and objective framework fusing different identification algorithm findings have yet to be made.

Encouraged by the lack of a unification perspective expressed above, this paper presents collocated deployments of different system identification methods on a numerical beam example for verification, and two large-scale testbeds for implementation. These are Frequency Domain Decomposition (FDD), Observer Kalman Filter Identification (OKID) using Eigensystem Realization Algorithm (ERA), Combined Deterministic-Stochastic Subspace Identification (D-SSI), System Realization Using Information Matrix (SRIM), and Autoregressive Exogenous Models (ARX). The first example is a numerical beam with three degree-of-freedom. The second one, Bridge 1, tested in the University of Nevada, Reno laboratories, and subjected progressive damage resulting from several shaking table tests. The third example, Bridge 2, includes field tests of a pedestrian link bridge connecting two multistory buildings. Based on the multi-output accelerometer readings from white noise and ambient vibration response measurements, modal parameters such as modal frequencies, mode shapes, and damping ratios are identified using FDD, OKID, D-SSI, SRIM, and ARX, and variation in identification results are investigated with crude quantified features on damage progression or intact states. Furthermore, a collaborative usage of multiple system identification methods is established as an improvement in identification confidence by systematically removing the outliers through a selfranking approach with quantified lack of agreement. Eventually, the other identification studies addressing the same benchmark bridges act for further assessment of varying features in terms of modal characteristics and their association with damage under environmental effects pertain under the synergistic findings from this study.

\section{System Identification Methods}

In this study, five different system/modal identification methods, such as FDD, OKID, D-SSI, SRIM, and ARX, are used. With the assumption that the input excitation is low-amplitude and broad-band, the system identification procedure can be considered as an output-only problem by setting the input vectors equal to zero where needed. The formulation of these identification methods is explained taking an $n$ degree-of-freedom system and $l$ numbers of time history data into consideration. The methods are classified according to the domains represented with FDD for the frequency domain, OKID, D-SSI, SRIM, and ARX for the time domain.

\subsection{Frequency domain system identification}

Frequency Domain Decomposition (FDD) (Brincker R. Z., 2001) is used to extract modal properties of the system using output-only measurements. Power spectral density matrix of the output $\boldsymbol{y}$ is decomposed by singular value decomposition as shown in Eq. (1)

$$
S_{y y}(w)=U(w) \Sigma(w) \mathrm{U}^{T}(w)
$$

where $\Sigma(w)$ is the diagonal matrix of singular values, $U(w)$ is the unitary matrix of singular vectors. By plotting the singular values as a function of frequency, the modal frequencies can be determined by picking the spectral peaks. What is more, the eigenvectors compose a set of square matrices spanning the frequency domain and the matrix corresponding to a modal frequency contains mode shape. In this way, modal frequencies and mode shapes can be identified with the output-only procedure. Moreover, using half-power bandwidth method or inverting the set of arrays from the frequency domain to the 
time domain, the decrement of the inverted time history contains the information regarding damping ratio, which is called Enhanced Frequency Domain Decomposition (EFDD) (Brincker R. V., 2001) (Jacobsen, 2006).

\subsection{Time-domain system identification methods}

Four different time-domain identification methods using multi-input multi-output measurements, including OKID/ERA, D-SSI, SRIM and ARX, are considered. The main idea of OKID approach (Phan, 1992) is to introduce a gain matrix into the state equation of a real system and make use of least square solutions to handle noisy data optimally. The approach turns OKID into a deterministic identification problem in which Markov parameters are obtained from an optimal steady-state observer. The combined D-SSI method (Van Overschee, 1994), (Van Overschee P., 1996) determines the non-steady Kalman filter state sequence directly from the input-output data using geometrical and linear algebra tools, e.g., orthogonal, oblique projections, and singular value decomposition. System matrices then are extracted from the estimated states and an extended observability matrix. The SRIM method (Juang J. N., 1997) identifies the system matrices directly from the input-output data by introducing a new system realization algorithm using data correlation concept and algebra manipulations. For system identification using ARX models, state-space matrices can be estimated via ARX coefficients. Representative mathematical formula of these four methods is detailed in the next sections. Herein, a general state-space system and Kalman filter observers are formulated as below.

Given input and output measurements, system matrices of a physical system can be identified through input-state-output relation at a discrete-time $k$ as follows

$$
\begin{gathered}
\boldsymbol{x}_{k+1}=\mathbf{A} \boldsymbol{x}_{k}+\mathbf{B} \boldsymbol{u}_{k}+\boldsymbol{p}_{k} \\
\boldsymbol{y}_{k}=\mathbf{C} \boldsymbol{x}_{k}+\mathbf{D} \boldsymbol{u}_{k}+\boldsymbol{m}_{k},
\end{gathered}
$$

where $\boldsymbol{x}_{k}$ is an $n \times 1$ state vector at time index $k(k=1: l), \boldsymbol{u}_{k}$ is an $r \times 1$ input vector ( $r$ inputs), $\boldsymbol{y}_{k}$ is an $m \times 1$ output vector ( $m$ sensors), $\boldsymbol{p}_{k}$ and $\boldsymbol{m}_{k}$ are assumed as zero-mean, white process and measurement noise, respectively, and uncorrelated with $\boldsymbol{u}_{k}$ and $\boldsymbol{y}_{k}$. System matrices A, B, C, and D are $n \times n$ system matrix, $n \times r$ input matrix, $m \times n$ output matrix, and $m \times r$ direct influence matrix, respectively.

Linear estimation of the real system, known as Kalman filter observer in non-steady states, can be performed in an innovation form as below

$$
\begin{aligned}
& \hat{\boldsymbol{x}}_{k+1}=\mathbf{A} \hat{\boldsymbol{x}}_{k}+\mathbf{B} \boldsymbol{u}_{k}+\mathbf{K}_{k} \boldsymbol{\varepsilon}_{k} \\
& \boldsymbol{y}_{k}=\mathbf{C} \hat{\boldsymbol{x}}_{k}+\mathbf{D} \boldsymbol{u}_{k}+\boldsymbol{\varepsilon}_{k}
\end{aligned}
$$

with $\boldsymbol{\varepsilon}_{k}$ is the estimation error of system output, $\boldsymbol{\varepsilon}_{k}=\boldsymbol{y}_{k}-\hat{\boldsymbol{y}}_{k}, \hat{\boldsymbol{x}}_{k}$ and $\hat{\boldsymbol{y}}_{\mathrm{k}}$ are observers of state and output, respectively. A Kalman gain matrix $\mathbf{K}$ is given by

$$
\begin{aligned}
& \mathbf{K}_{k}=\left(\mathbf{A} \Pi_{k} \mathbf{C}^{\mathrm{T}}+\mathbf{R}_{p m}\right)\left(\mathbf{R}_{m m}+\mathbf{C} \Pi_{k} \mathbf{C}^{\mathrm{T}}\right)^{-1} \\
& \Pi_{k+1}=\mathbf{A} \Pi_{k} \mathbf{A}^{\mathrm{T}}-\left(\mathbf{A} \Pi_{k} \mathbf{C}^{\mathrm{T}}+\mathbf{R}_{p m}\right)\left(\mathbf{R}_{m m}+\mathbf{C} \Pi_{k} \mathbf{C}^{\mathrm{T}}\right)^{-1}\left(\mathbf{A} \Pi_{k} \mathbf{C}^{\mathrm{T}}+\mathbf{R}_{p m}\right)^{\mathrm{T}}+\mathbf{R}_{p p},
\end{aligned}
$$

where $\mathbf{R}_{p p}$ and $\mathbf{R}_{m m}$ are covariance matrices of process noise $\boldsymbol{p}_{k}$ and measurement noise $\boldsymbol{m}_{k}$, respectively, $\mathbf{R}_{p m}$ is a cross-covariance matrix of $\boldsymbol{p}_{k}$ and $\boldsymbol{m}_{k}$. Equation (7) represents the covariance matrix of state estimation error, known as the Riccati equation. Following sections describe four identification methods which may require different dynamic performances of the system. 


\subsubsection{Observer Kalman Filter Identification Using ERA}

Assuming that the process noise $\boldsymbol{p}_{k}$ and measurement noise $\boldsymbol{m}_{k}$ are stationary, mutually uncorrelated, and time step $k \rightarrow \infty$, Eq. (4) and (5) become linear time-invariant Kalman filter observers in a steadystate;

$$
\begin{aligned}
& \hat{\boldsymbol{x}}_{k+1}=\mathbf{G} \hat{\boldsymbol{x}}_{k}+\mathbf{H} \boldsymbol{u}_{k}+\mathbf{K} \boldsymbol{y}_{k} \\
& \boldsymbol{y}_{k}=\mathbf{C} \hat{\boldsymbol{x}}_{k}+\mathbf{D} \boldsymbol{u}_{k}+\boldsymbol{\varepsilon}_{k}
\end{aligned}
$$

where $\mathbf{G}, \mathbf{H}$, and $\mathbf{K}$ are matrices independent with time index $k$, given by

$$
\begin{aligned}
& \mathbf{G}=\mathbf{A}-\mathbf{K C}, \\
& \mathbf{H}=\mathbf{B}-\mathbf{K D}, \\
& \mathbf{K}=\mathbf{A} \Pi \mathbf{C}^{\mathbf{T}}\left(\mathbf{R}_{m m}+\mathbf{C} \Pi \mathbf{C}^{\mathbf{T}}\right)^{-1}, \\
& \boldsymbol{\Pi}=\mathbf{A} \Pi \mathbf{A}^{\mathbf{T}}-\mathbf{A} \Pi \mathbf{C}^{\mathbf{T}}\left(\mathbf{R}_{m m}+\mathbf{C} \Pi \mathbf{C}^{\mathbf{T}}\right)^{-1} \mathbf{C} \Pi \mathbf{A}^{\mathbf{T}}+\mathbf{R}_{p p},
\end{aligned}
$$

Equation (9) can be written in a matrix form with considering time step $k \geq p$, with $p$ chosen sufficiently large $(p>>n)$ to ensure the Kalman filter in the steady-state

$$
\mathbf{Y}=\Psi \mathbf{W}+\mathbf{E}
$$

where $\mathbf{Y}=\left[\boldsymbol{y}_{p}, \boldsymbol{y}_{p+1}, \ldots, \boldsymbol{y}_{l-1}\right], \mathbf{W}=\left[\boldsymbol{w}_{p}, \boldsymbol{w}_{p+1}, \ldots, \boldsymbol{w}_{l-1}\right]$ with $\mathbf{w}_{k}=\left[\boldsymbol{u}_{k} ; \boldsymbol{u}_{k-1} ; \boldsymbol{y}_{k-1} ; \ldots ; \boldsymbol{u}_{k-p} ; \boldsymbol{y}_{k-p}\right], \boldsymbol{\Psi}=[\mathbf{D}, \mathbf{C P}$, $\left.\mathbf{C G P}, \ldots, \mathbf{C G}^{p-2} \mathbf{P}, \mathbf{C G}^{p-1} \mathbf{P}\right]$ with $\mathbf{P}=[\mathbf{H}, \mathbf{K}]$, and $\mathbf{E}=\left[\boldsymbol{\varepsilon}_{p}, \boldsymbol{\varepsilon}_{p+1}, \ldots, \boldsymbol{\varepsilon}_{l-1}\right]$.

Solving Eq. (14) by the Least Square (LS) method yields $\hat{\boldsymbol{\Psi}}=\mathbf{Y} \mathbf{W}^{\mathrm{T}}\left(\mathbf{W} \mathbf{W}^{\mathrm{T}}\right)^{-1}$, where $\hat{\mathbf{\Psi}}$ is the Markov parameter sequence of the Kalman filter observer. The direct influence matrix $\mathbf{D}$ can be identified by the first partition $\hat{\mathbf{\Psi}}_{0}$ of $\hat{\mathbf{\Psi}}$ as shown in $\hat{\Psi}=\left[\hat{\Psi}_{0}, \hat{\Psi}_{1}, \hat{\Psi}_{2}, \ldots, \hat{\Psi}_{p}\right]$. Other system Markov parameters $\mathrm{CA}^{k-}$

${ }^{1} \mathbf{B}$ and observer gain Markov parameters $\mathbf{C A}^{k-1} \mathbf{K}(k=1,2,3 \ldots)$ can be recovered from $\hat{\Psi}_{1}, \hat{\Psi}_{2}, \ldots, \hat{\Psi}_{p}$ , where the recovery process is detailed in (Juang J. N., 1993). Finally, the identification process can be completed by determining A, B, C, and $\mathbf{K}$ from the Markov parameters by ERA (Juang J. N., 1985).

\subsubsection{Combined Deterministic-Stochastic System Identification (D-SSI)}

With some algebraic manipulations, Eq. (2) and (3) can be rewritten in matrix forms as follows

$$
\begin{aligned}
& \mathbf{Y}_{0 \mid i-1}=\mathbf{O}_{i} \mathbf{X}_{0}^{d}+\mathbf{T}_{i}^{d} \mathbf{U}_{0 \mid i-1}+\mathbf{E}_{\alpha i-1}^{s} \\
& \mathbf{Y}_{i \mid 2 i-1}=\mathbf{O}_{i} \mathbf{X}_{i}^{d}+\mathbf{T}_{i}^{d} \mathbf{U}_{i \mid 2 i-1}+\mathbf{E}_{\mid 2 i-1}^{s}
\end{aligned}
$$

where $\mathbf{X}_{0}^{d}=\left[\boldsymbol{x}_{0}, \boldsymbol{x}_{1}, \ldots, \boldsymbol{x}_{j-1}\right]^{n \times j}, \mathbf{X}_{i}^{d}=\left[\boldsymbol{x}_{i}, \boldsymbol{x}_{i+1}, \ldots, \boldsymbol{x}_{i+j-1}\right]^{n \times j}$ are non-steady Kalman filter state sequences, $\mathbf{E}_{0 \mid i-1}^{s}$ and $\mathbf{E}_{i \mid 2 i-1}^{s}$ are $i m \times j$ measurement noise matrices, $\mathbf{O}_{i}=\left[\mathbf{C} ; \mathbf{C A} ; \mathbf{C A}^{2} ; \ldots ; \mathbf{C A}^{i-1}\right]^{i m \times n}$ is an extended observability matrix $(i>n)$, and $\mathbf{T}_{i}^{d}$ is a Toeplitz matrix, 


$$
\mathbf{T}_{i}^{d}=\left[\begin{array}{ccccc}
\mathbf{D} & 0 & 0 & \ldots & 0 \\
\mathbf{C B} & \mathbf{D} & 0 & \ldots & 0 \\
\mathbf{C A B} & \mathbf{C B} & \mathbf{D} & \ldots & 0 \\
\vdots & \vdots & \vdots & \vdots & \vdots \\
\mathbf{C A}^{i-2} \mathbf{B} & \mathbf{C A}^{i-3} \mathbf{B} & \mathbf{C A}^{i-4} \mathbf{B} & \ldots & \mathbf{D}
\end{array}\right]^{i m i r}
$$

and Block Hankel matrices of input and output measurements are respectively defined as follows

$$
\mathbf{U}_{0 \mid i-1}=\left[\begin{array}{ccccc}
u_{0} & u_{1} & u_{2} & \ldots & u_{j-1} \\
u_{1} & u_{2} & u_{3} & \ldots & u_{j} \\
\vdots & \vdots & \vdots & \vdots & \vdots \\
u_{i-1} & u_{i} & u_{i+1} & \ldots & u_{i+j-2}
\end{array}\right]^{i r \times j}, \mathbf{Y}_{0 \mid i-1}=\left[\begin{array}{ccccc}
y_{0} & y_{1} & y_{2} & \ldots & y_{j-1} \\
y_{1} & y_{2} & y_{3} & \ldots & y_{j} \\
\vdots & \vdots & \vdots & \vdots & \vdots \\
y_{i-1} & y_{i} & y_{i+1} & \ldots & y_{i+j-2}
\end{array}\right]^{i m \times j}
$$

where $i$ is the block row number defined by users $(i>n)$ and $j(j=l-2 i+1)$ covering the entire length $l$ of data. Similarly, $\mathbf{U}_{0 \mid i}, \mathbf{Y}_{0 \mid i}, \mathbf{U}_{i \mid 2 i-1}, \mathbf{Y}_{i \mid 2 i-1}, \mathbf{U}_{i+1 \mid 2 i-1}, \mathbf{Y}_{i+1 \mid 2 i-1}$ are defined in the same manner as Eq. (18).

To determine system matrices which will be shown later, the non-steady Kalman filter observers of the system described in Eq. (4) and (5) can be rewritten under following matrix form

$$
\left[\begin{array}{c}
\hat{\mathbf{X}}_{i+1} \\
\mathbf{Y}_{i \mid i}
\end{array}\right]=\left[\begin{array}{cc}
\mathbf{A} & \mathbf{B} \\
\mathbf{C} & \mathbf{D}
\end{array}\right]\left[\begin{array}{c}
\hat{\mathbf{X}}_{i} \\
\mathbf{U}_{i \mid i}
\end{array}\right]+\left[\begin{array}{c}
e_{1} \\
e_{2}
\end{array}\right],
$$

where $e_{1}$ and $e_{2}$ are residuals of the state sequence and system output, $\mathbf{U}_{i \mid i}$ and $\mathbf{Y}_{i \mid i}$ are the $i^{\text {th }}$ block row matrices of input and output measurements, respectively. Herein, oblique and orthogonal projections are introduced. These projections are useful to interpret the relation between the state sequence, system matrices and input/output data

$$
\begin{aligned}
& \mathbf{L}_{i}=\mathbf{Y}_{i \mid 2 i-1} / \mathbf{U}_{i \mid 2 i-1} \mathbf{W}_{0 \mid i-1}, \\
& \mathbf{Z}_{i}=\mathbf{Y}_{i \mid 2 i-1} /\left[\begin{array}{l}
\mathbf{W}_{0 \mid i-1} \\
\mathbf{U}_{i \mid 2 i-1}
\end{array}\right]_{\text {with }} \mathbf{W}_{0 \mid i-1}=\left[\begin{array}{l}
\mathbf{U}_{0 \mid i-1} \\
\mathbf{Y}_{0 \mid i-1}
\end{array}\right], \\
& \mathbf{Z}_{i+1}=\mathbf{Y}_{i+1 \mid 2 i-1} /\left[\begin{array}{l}
\mathbf{W}_{i+1 \mid 2 i-1} \\
\mathbf{U}_{i+1 \mid 2 i-1}
\end{array}\right] \text { with } \mathbf{W}_{i+|| 2 i-1}=\left[\begin{array}{l}
\mathbf{U}_{i+1 \mid 2 i-1} \\
\mathbf{Y}_{i+1 \mid 2 i-1}
\end{array}\right] .
\end{aligned}
$$

By taking Singular Value Decomposition (SVD) of weighted oblique the projection, $\mathbf{L}_{i} / \mathbf{U}_{i \mid 2 i-1}^{\perp}$,

$$
\mathbf{L}_{i} / \mathbf{U}_{i \mid 2 i-1}^{\perp}=\left[\mathbf{U}_{1}, \mathbf{U}_{2}\right]\left[\begin{array}{cc}
\mathbf{S}_{1} & 0 \\
0 & 0
\end{array}\right]\left[\begin{array}{l}
\mathbf{V}_{1} \\
\mathbf{V}_{2}
\end{array}\right],
$$


the system order and the extended observability matrix $\mathbf{O}_{i}$ can be determined. The system order theoretically equals to the number of non-zero singular values of the diagonal matrix $\mathbf{S}_{1}$ and $\mathbf{O}_{i}=\mathbf{U}_{1} \mathbf{S}_{1}^{1 / 2}$. Hereafter, system matrices $\mathbf{A}, \mathbf{B}, \mathbf{C}$, and $\mathbf{D}$ can be identified using the estimated states and/or the extended observability matrix $\mathbf{O}_{i}$ as presented in two following algorithms.

Algorithm 1: Following the Theorem 12 in (Van Overschee P., 1996), the non-steady state Kalman filter state sequence can be estimated directly from the input-output data without known system matrices, $\hat{\mathbf{X}}_{i}=\mathbf{O}_{i}^{\dagger} \mathbf{L}_{i}$, where $(.)^{\dagger}$ symbolizes for the Moore-Penrose pseudo-inverse. A similar procedure is also applied for $\hat{\mathbf{X}}_{i+1}=\mathbf{O}_{i+1}^{\dagger} \mathbf{L}_{i+1}$. System matrices can be identified from Eq. (19) by the LS method given the estimated state sequences, and further details can be found in Chapter 4 (Van Overschee P., 1996). When one of the following conditions, such as $i \rightarrow \infty$, purely deterministic system or white noise input is not satisfied, LS solutions will produce biased estimates of the system matrices.

Algorithm 2: Another way to determine system matrices is by using the extended observability matrix $\mathbf{O}_{i}$ resulted from SVD of $\mathbf{Z}_{i}$, which will be applied in this paper. Equation (19) can be rewritten as a set of linear equations by hiding all unknown states following Theorem 11 in (Van Overschee P., 1996)

$$
\left[\begin{array}{c}
\mathbf{O}_{i-1}^{\dagger} \mathbf{Z}_{i+1} \\
\mathbf{Y}_{i \mid i}
\end{array}\right]=\left[\begin{array}{c}
\mathbf{A} \\
\mathbf{C}
\end{array}\right] \mathbf{O}_{i}^{\dagger} \mathbf{Z}_{i}+\boldsymbol{\Lambda} \mathbf{U}_{i \mid 2 i-1}+\left[\begin{array}{l}
e_{1} \\
e_{2}
\end{array}\right]
$$

where $\boldsymbol{\Lambda}=\left[\begin{array}{c}\left(\mathbf{B} \mid \mathbf{O}_{i-1}^{\dagger} \cdot \mathbf{T}_{i-1}^{d}\right)-\mathbf{A} \cdot \mathbf{O}_{i}^{\dagger} \cdot \mathbf{T}_{i}^{d} \\ (\mathbf{D} \mid 0)-\mathbf{C} \cdot \mathbf{O}_{i}^{\dagger} \cdot \mathbf{T}_{i}^{d}\end{array}\right]$

System matrices $\mathbf{A}$ and $\mathbf{C}$ can be estimated by solving Eq. (24) with the LS method. To increase the accuracy of the estimates, the observability matrices $\mathbf{O}_{i}^{\dagger}$ and $\mathbf{O}_{i-1}^{\dagger}$ can be recomputed from known $\mathbf{A}$ and $\mathbf{C}$ matrices. Besides, matrices $\mathbf{B}$ and $\mathbf{D}$ can be then determined by the minimization of residuals in Eq.(24). By avoiding the use of non-steady Kalman filter observers, Algorithm 2 can be considered as a robust identification method in practice, e.g., finite measurement and non-white noise inputs.

\subsubsection{System Realization Using Information Matrix (SRIM)}

A new realization algorithm SRIM using data correlation concept of shifted input and output (Juang J. N., 1997) is introduced to identify system matrices directly from data. Using the previously defined matrices in section 2.2.1, the input-state-output relation in Eq. (2) and (3) can be performed for each time index $k(k=0,1,2, \ldots, l)$ in a matrix form as follows:

$$
\mathbf{Y}_{k}=\mathbf{O} \mathbf{X}_{k}+\mathbf{T U}_{k}+\mathbf{E}_{k}
$$

where definitions of $\mathbf{O}, \mathbf{T}$, and $\mathbf{E}_{k}$ are like those of $\mathbf{O}_{i}, \mathbf{T}_{i}{ }^{d,}$ and $\mathbf{E}_{0 \mid i-1}^{s}$ in Eq. (15), with $i$ integer defined by users $(i>n / m+1), n$ is the desired system order. The state matrix $\mathbf{X}_{k}=\left[\boldsymbol{x}_{k}, \boldsymbol{x}_{k+1}, \ldots, \boldsymbol{x}_{k+j-1}\right]^{n \times j}$ with integer $j$ chosen such that $j=l-k-i+2$ covering the data length $l$, 


$$
\mathbf{U}_{i}(k)=\left[\begin{array}{ccccc}
\boldsymbol{u}_{k} & \boldsymbol{u}_{k+1} & \boldsymbol{u}_{k+2} & \ldots & \boldsymbol{u}_{k+j-1} \\
\boldsymbol{u}_{k+1} & \boldsymbol{u}_{k+2} & \boldsymbol{u}_{k+3} & \ldots & \boldsymbol{u}_{k+j} \\
\vdots & \vdots & \vdots & \vdots & \vdots \\
\boldsymbol{u}_{k+i-1} & \boldsymbol{u}_{k+i} & \boldsymbol{u}_{k+i+1} & \ldots & \boldsymbol{u}_{k+i+j-2}
\end{array}\right]^{i r \times j} \text { and } \mathbf{Y}_{i}(k)=\left[\begin{array}{ccccc}
\boldsymbol{y}_{k} & \boldsymbol{y}_{k+1} & \boldsymbol{y}_{k+2} & \ldots & \boldsymbol{y}_{k+j-1} \\
\boldsymbol{y}_{k+1} & \boldsymbol{y}_{k+2} & \boldsymbol{y}_{k+3} & \ldots & \boldsymbol{y}_{k+j} \\
\vdots & \vdots & \vdots & \vdots & \vdots \\
\boldsymbol{y}_{k+i-1} & \boldsymbol{y}_{k+i} & \boldsymbol{y}_{k+i+1} & \ldots & \boldsymbol{y}_{k+i+j-2}
\end{array}\right]^{i m \times j}
$$

To determine system matrices $\mathbf{A}, \mathbf{C}$ from $\mathbf{O}$ and $\mathbf{B}, \mathbf{D}$ from $\mathbf{T}$, following autocorrelation and crosscorrelation matrices are used to eliminate either $\mathbf{T}$ or $\mathbf{O}$ from Eq. (25)

$$
\begin{aligned}
& \mathbf{R}_{\mathbf{y y}}=(1 / j) \mathbf{Y}_{k} \mathbf{Y}_{k}^{\mathrm{T}}, \mathbf{R}_{\mathbf{u u}}=(1 / j) \mathbf{U}_{k} \mathbf{U}_{k}^{\mathrm{T}}, \mathbf{R}_{\mathbf{x x}}=(1 / j) \mathbf{X}_{k} \mathbf{X}_{k}^{\mathrm{T}} \\
& \mathbf{R}_{\mathbf{y u}}=(1 / j) \mathrm{Y}_{k} \mathrm{U}_{k}^{\mathrm{T}}, \mathbf{R}_{\mathbf{y x}}=(1 / j) \mathrm{Y}_{k} \mathrm{X}_{k}^{\mathrm{T}}, \mathbf{R}_{\mathbf{x u}}=(1 / j) \mathrm{X}_{k} \mathrm{U}_{k}^{\mathrm{T}}
\end{aligned}
$$

Post-multiplying both sides of Eq. (25) by $(1 / j) \mathbf{U}_{i}^{\mathrm{T}},(1 / j) \mathbf{Y}_{i}^{\mathrm{T}}$, and $(1 / j) \mathbf{X}_{i}^{\mathrm{T}}$ yields Eq. (27), (28), and (29), respectively

$$
\begin{aligned}
& \mathbf{R}_{\mathbf{y u}}=\mathbf{O R}_{\mathbf{x u}}+\mathbf{T R}_{\mathbf{u u}} \\
& \mathbf{R}_{\mathbf{y y}}=\mathbf{O R}_{\mathbf{y x}}+\mathbf{T R}_{\mathbf{y u}}^{\mathrm{T}} \\
& \mathbf{R}_{\mathbf{y x}}=\mathbf{O R}_{\mathbf{x x}}+\mathbf{T R}_{\mathbf{x u}}^{\mathrm{T}} .
\end{aligned}
$$

Substituting $\mathbf{T}=\left(\mathbf{R}_{\mathbf{y u}}-\mathbf{O} \mathbf{R}_{\mathbf{x u}}\right) \mathbf{R}^{-1}$ uu (if $\left.\mathbf{R}_{\mathbf{u u}}>0\right)$ deduced from Eq. (27) into Eq. (28) and (29) returns

$$
\overline{\mathbf{R}}_{\mathbf{y y}}=\mathbf{O} \overline{\mathbf{R}}_{\mathbf{x x}} \mathbf{O}^{\mathrm{T}}
$$

where $\overline{\mathbf{R}}_{\mathrm{yy}}=\mathbf{R}_{\mathrm{yy}}-\mathbf{R}_{\mathrm{yu}} \mathbf{R}_{\mathrm{uu}}^{-1} \mathbf{R}_{\mathrm{yu}}^{T}$,

$$
\overline{\mathbf{R}}_{\mathrm{xx}}=\mathbf{R}_{\mathrm{xx}}-\mathbf{R}_{\mathrm{xu}} \mathbf{R}_{\mathrm{uu}}^{-1} \mathbf{R}_{\mathrm{xu}}^{T} .
$$

Then, taking Singular Value Decomposition of the $i m \times i m$ matrix $\overline{\mathbf{R}}_{\mathbf{y y}}$ yields

$$
\overline{\mathbf{R}}_{\mathbf{y y}}=\left[\mathbf{U}_{1}, \mathbf{U}_{2}\right]\left[\begin{array}{ll}
\mathbf{S} & 0 \\
0 & 0
\end{array}\right]\left[\begin{array}{l}
\mathbf{U}_{1}^{T} \\
\mathbf{U}_{2}^{T}
\end{array}\right]=\mathbf{U}_{1} \mathbf{S} \mathbf{U}_{1}^{T} \text {. }
$$

Equation (30) and (33) imply $\mathbf{O}=\mathbf{U}_{1}$. With $\mathbf{O}$ known, matrices $\mathbf{A}$ and $\mathbf{C}$ can be easily identified from the observability matrix $\mathbf{O}=\left[\mathbf{C} ; \mathbf{C A} ; \mathbf{C A}^{2} ; \ldots ; \mathbf{C A}^{i-1}\right]^{i m \times n}$. To determine $\mathbf{B}$ and $\mathbf{D}$ from $\mathbf{T}$, the matrix $\mathbf{O}$ should be eliminated from Eq. (25). Post-multiplying Eq. (25) by the $i m \times n$ matrix $\mathbf{U}_{1}{ }^{\mathrm{T}}$ and utilizing orthogonality property of $\mathbf{U}_{1}\left(\mathbf{U}_{1}=\mathbf{O}\right)$ and $\mathbf{U}_{2}$ produces

$$
\mathbf{T}=\mathbf{R}_{\mathrm{yu}} \mathbf{R}_{\mathrm{uu}}^{-1}
$$

Based on the previous definition of Toeplitz matrix $\mathbf{T}$ in Eq. (17), one can partition $\mathbf{T}$ into parts to extract matrices $\mathbf{B}$ and $\mathbf{D}$.

\subsubsection{Auto-regressive Exogenous Models (ARX)}

The input-output relationship can be approximated using auto-regressive time series with exogenous inputs, called ARX model. The ARX model represents the simple linear input-output structure with two main parts, an auto-regressive part and an exogenous input part to predict the future response $y_{k}$ from past measurements, as presented in the following equation: 


$$
\boldsymbol{y}_{k}+\boldsymbol{\alpha}_{1} \boldsymbol{y}_{k-1}+\boldsymbol{\alpha}_{2} \boldsymbol{y}_{k-2}+\ldots+\boldsymbol{\alpha}_{p} \boldsymbol{y}_{k-p}=\boldsymbol{\beta}_{0} \boldsymbol{u}_{k}+\boldsymbol{\beta}_{1} \boldsymbol{u}_{k-1}+\boldsymbol{\beta}_{2} \boldsymbol{u}_{k-2}+\ldots+\boldsymbol{\beta}_{p} \boldsymbol{u}_{k-p}+\boldsymbol{e}_{k}
$$

where $\boldsymbol{\alpha}_{i}(m \times m)$ and $\boldsymbol{\beta}_{i}(m \times r)$ are constant coefficient matrices, with $i=1,2, \ldots, p$ and $p$ is an ARX order chosen by users. The term $\mathbf{e}_{k}$ accounts for the disturbance factor from modelling errors and measurement noise. The equattion (35) can be rewrited in the following matrix form:

$$
\mathbf{Y}=\mathbf{\Psi V}+\mathbf{E},
$$

$$
\text { where, input matrix } \mathbf{V}=\left[\begin{array}{ccccc}
\boldsymbol{u}_{p} & \boldsymbol{u}_{p+1} & \boldsymbol{u}_{p+2} & \cdots & \boldsymbol{u}_{l} \\
\boldsymbol{u}_{p-1} & \boldsymbol{u}_{p} & \boldsymbol{u}_{p+1} & \cdots & \boldsymbol{u}_{l-1} \\
\boldsymbol{y}_{p-1} & \boldsymbol{y}_{p} & \boldsymbol{y}_{p+1} & \cdots & \boldsymbol{y}_{l-1} \\
\boldsymbol{u}_{p-2} & \boldsymbol{u}_{p-1} & \boldsymbol{u}_{p} & \cdots & \boldsymbol{u}_{l-2} \\
\boldsymbol{y}_{p-2} & \boldsymbol{y}_{p-1} & \boldsymbol{y}_{p} & \cdots & \boldsymbol{y}_{l-2} \\
\vdots & \vdots & \vdots & \cdots & \vdots \\
\boldsymbol{u}_{0} & \boldsymbol{u}_{1} & \boldsymbol{u}_{2} & \cdots & \boldsymbol{u}_{l-p} \\
\boldsymbol{y}_{0} & \boldsymbol{y}_{1} & \boldsymbol{y}_{2} & \cdots & \boldsymbol{y}_{l-p}
\end{array}\right]_{(p(m+r)+r) \times(l-p+1)}
$$

output matrix $\mathbf{Y}=\left[\boldsymbol{y}_{p}, \boldsymbol{y}_{p+1}, \ldots, \boldsymbol{y}_{l}\right]_{m \times(l-p+1)}$, coefficient matrix $\boldsymbol{\Psi}=\left[\boldsymbol{\beta}_{0}, \boldsymbol{\beta}_{1},-\boldsymbol{\alpha}_{1}, \ldots, \boldsymbol{\beta}_{p},-\boldsymbol{\alpha}_{p}\right]_{m \times(p(m+r)+r)}$ and $\mathbf{E}=\left[\boldsymbol{e}_{p}, \boldsymbol{e}_{p+1}, \ldots, \boldsymbol{e}_{l}\right]_{m \times(l-p+1)}$. The coefficient matrix can be estimated by Least Square, and used for calculating system matrices, $\mathbf{A}, \mathbf{B}, \mathbf{C}$ and $\mathbf{D}$ :

$$
\begin{aligned}
\mathbf{A} & =\left[\begin{array}{ccccc}
\mathbf{0} & \mathbf{0} & \cdots & \mathbf{0} & -\boldsymbol{\alpha}_{p} \\
\mathbf{I} & \mathbf{0} & \cdots & \mathbf{0} & -\boldsymbol{\alpha}_{p-1} \\
\mathbf{0} & \mathbf{I} & \cdots & \mathbf{0} & -\boldsymbol{\alpha}_{p-2} \\
\vdots & \vdots & \cdots & \vdots & \vdots \\
\mathbf{0} & \mathbf{0} & \cdots & \mathbf{I} & -\boldsymbol{\alpha}_{1}
\end{array}\right]_{m p \times m p}, \\
\mathbf{B} & =\left[\begin{array}{llllll}
\boldsymbol{\beta}_{p}-\boldsymbol{\alpha}_{p} \boldsymbol{\beta}_{0}, & \boldsymbol{\beta}_{p-1}-\boldsymbol{\alpha}_{p-1} \boldsymbol{\beta}_{0}, \quad \boldsymbol{\beta}_{p-2}-\boldsymbol{\alpha}_{p-2} \boldsymbol{\beta}_{0} & \cdots & \boldsymbol{\beta}_{1}-\boldsymbol{\alpha}_{1} \boldsymbol{\beta}_{0}
\end{array}\right]_{m p \times r}, \\
\mathbf{C} & =\left[\begin{array}{lllll}
\mathbf{0}, & \mathbf{0}, & \mathbf{0} & \cdots & \mathbf{I}
\end{array}\right]_{m \times m p}, \text { and } \mathbf{D}=\boldsymbol{\beta}_{o} .
\end{aligned}
$$

Modal parameters can be then determined from those state-space matrices by eigenvalues and eigenvectors as presented in the next section.

\subsubsection{Modal Parameter Identification from State-space Matrices}

The modal parameters as frequencies, damping ratios, and mode shapes from above time-domain SI methods can be identified from the state-space matrices $\mathbf{A}$ and $\mathbf{C}$. The system matrix $\mathbf{A}$ can be decomposed into eigenvectors and eigenvalues as follows:

$$
\mathbf{A}=\mathbf{V S V}^{-1},
$$

with $\mathbf{S}(n \times n)$ is a diagonal matrix containing eigenvalues $\mu_{i}$ and $\mathbf{V}(n \times n)$ is an eigenvector matrix. The eigenfrequency $f_{i}$, damping ratio $\xi_{i}$ and mode shape $\phi_{i}$ are then determined by

$$
\begin{aligned}
& f_{i}=\ln \left(\mu_{i}\right) / \mathrm{dt}, \\
& \xi_{i}=-\mu_{i}^{r} /\left|\mu_{i}\right|,
\end{aligned}
$$

and

$$
\phi_{i}=\mathbf{C V} \text {, }
$$


with $\mu_{i}^{r}$ is the real part of a complex number and $\left|\mu_{i}\right|$ is a complex modulus.

The time-domain system identification procedures determining modal parameters depend on the order of selection. Singular values as a function of system order indicate the proper order where a sudden value drop is observed. However, in extreme noise cases, such a drop may not be prevalent, and further steps may need to be pursued, such as a stabilization diagram for accurate identification. The stabilization diagram reveals the repetitiveness of modal behaviour at a series of different system orders, and stable poles can be distinguished based on the consistency over the domain.

\subsubsection{Stabilization Diagram and Model Order Selection}

Modal identification with parametric methods is prone to non-physical modal findings, which need to be discarded through stabilization diagrams. When modal identification is conducted in consecutive system orders, stability criteria can quantify pole stability and visualize distinction of physical modes from mathematical ones via vertical lines. The stabilization criteria for frequency, damping ratio, and mode shape is formulated as:

$$
\begin{aligned}
& \frac{f_{i+1}-f_{i}}{f_{i}}<\lim _{f}(\%) \\
& \frac{\xi_{i+1}-\xi_{i}}{\xi_{i}}<\lim _{\xi}(\%) \\
& 1-M A C_{i, i+1}=1-\frac{\left|\phi_{i}^{T} \phi_{i+1}\right|^{2}}{\left(\phi_{i}^{T} \phi_{i}\right)\left(\phi_{i+1}^{T} \phi_{i+1}\right)}<\lim _{M A C}(\%)
\end{aligned}
$$

where $i$ and $i+1$ are consecutive system orders, $f$ is frequency, $\xi$ is damping ratio, $\phi$ is mode shape, $(.)^{T}$ is a complex conjugate transpose, $\lim _{f}, \lim _{\xi}$, and $\lim _{M A C}$ are limits defined as 0.01, 0.05, and 0.05, respectively. By default, this study uses $0.01,0.05$, and 0.05 limit measures associated with frequency, damping ratio, and $1-M A C$, respectively.

\subsubsection{Collection of Method Characteristics for the Synergistic Combination}

From the methodologies expressed above, it can be observed that many features of the proposed algorithms are discovered in the literature, which can serve for judgement of the method performances under the synergistic scheme. Table 1 summarizes the existing literature's observations denoting the characteristics, weaknesses, and strengths of each method referred by former studies. An outcome of such an evaluation can guide expert decisions if one method is superior to others; however, one should note that such a process might possess a qualitative and subjective nature. For example, according to the authors' opinion, D-SSI might be more favourable with least penalties over alternatives, but another expert may disagree with doing so. A more systematic approach to prioritize methods' weights on the synergistic process requires a quantitative framework, which is discussed further in Section 7. 


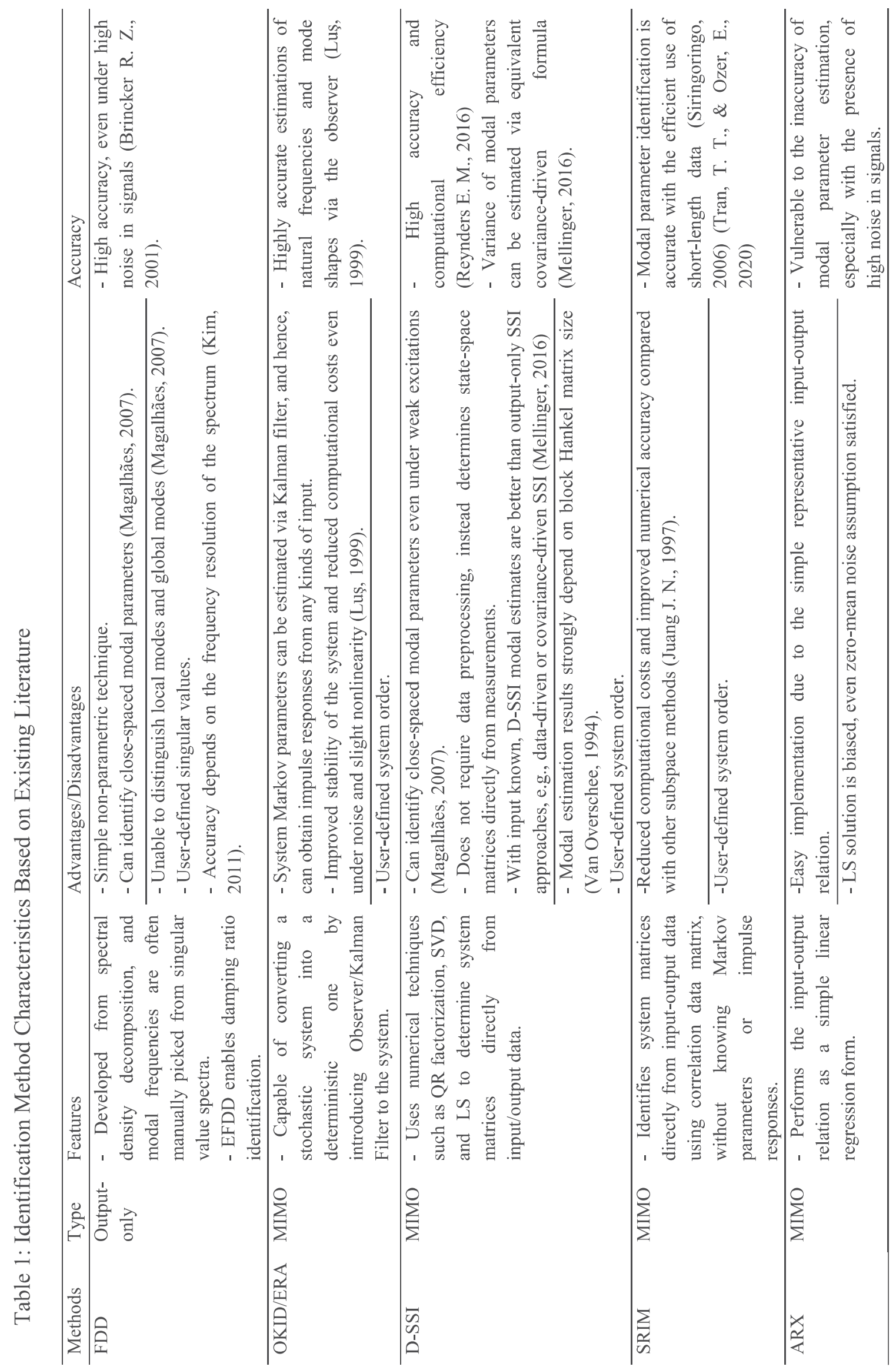




\section{Numerical Example of a Single-Span Beam}

In this section, a proof-of-concept application is performed on a single-span beam structure simulated numerically. A 12-meter-long beam supported with fixed and pinned ends has three lumped masses along the length. Therefore, the structure represents a 3-degree-of-freedom (3DOF) system and is subjected to white noise excitation at supports in the vertical direction. The beam mechanical properties are given as $\mathrm{E}=25 \times 10^{6} \mathrm{MPa}, \mathrm{L}=12 \mathrm{~m}, \mathrm{I}=0.3 \mathrm{~m}^{4}, \mathrm{~m}=91.8 \mathrm{t}$, $\xi=0.02, v=0.2$ and the layout is given in Figure 1.

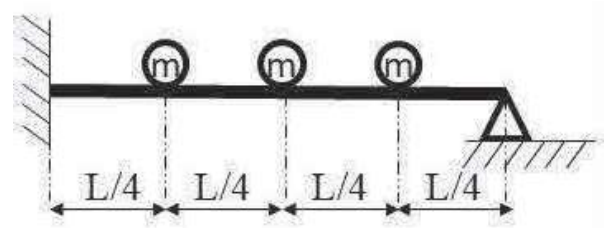

Figure 1. Numerical beam example's layout and characteristics.

The white noise excitation reaches an absolute maximum amplitude of $0.01 \mathrm{~g}$, and accordingly, the nodal responses are simulated based on the parameters expressed above. Figure 2 shows the acceleration response time histories and spectral characteristics of each node's vibration. For the given features, the first, second, and third modal frequencies and mode shapes can be derived from the stiffness and mass matrices' eigenvalues and eigenvectors as a reference set. $2 \%$ modal damping ratio applies to all three vibration modes.
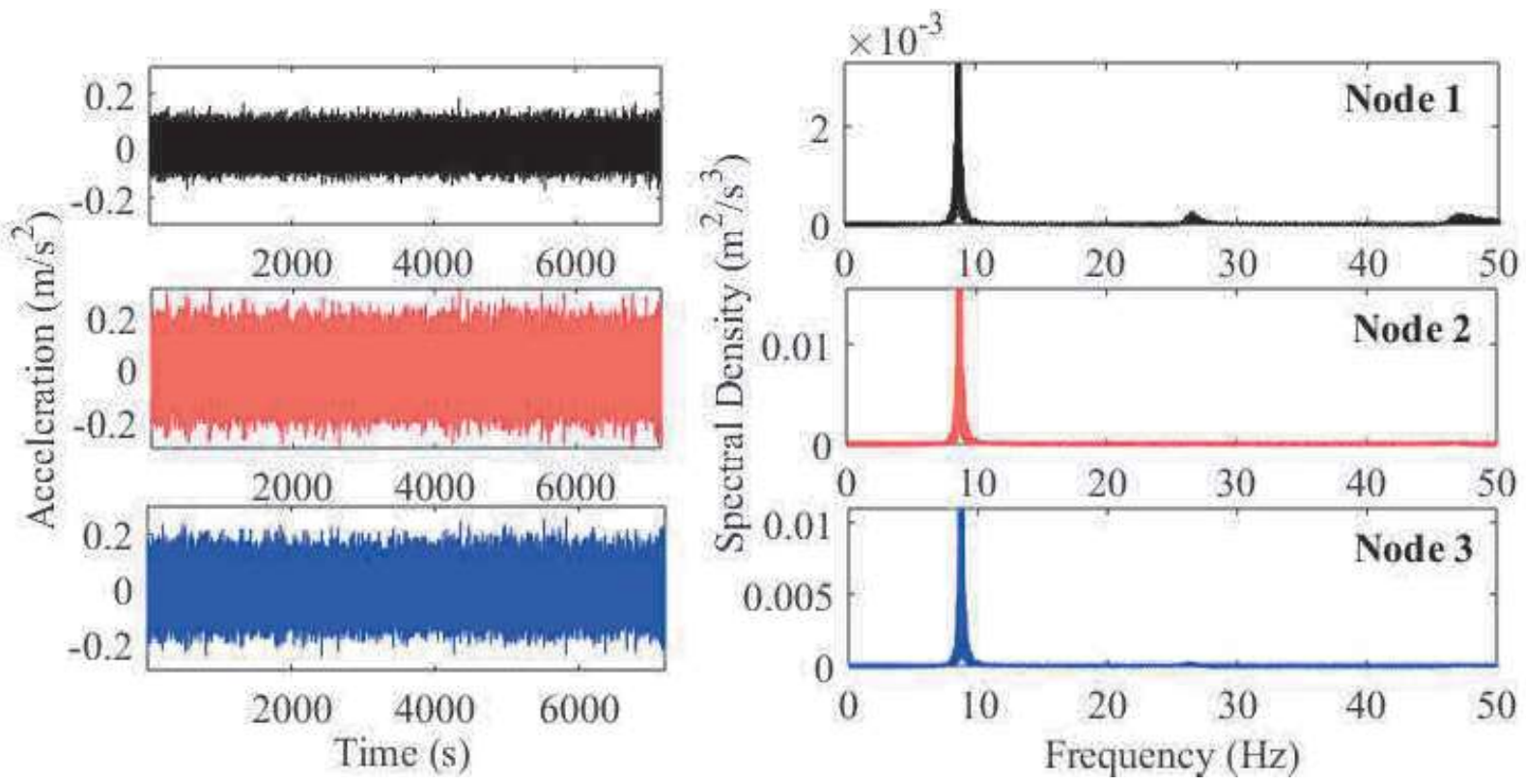

Figure 2. Acceleration time history and Fourier spectra at Node 1, 2, and 3.

The simulated response is then used for modal analysis following each identification method. Figure 3 shows stabilization diagrams for parametric methods distinguishing physical poles from the mathematical ones through the stabilization criteria mentioned in the previous section. As a result, together with the actual values, the modal frequencies and damping ratios are identified as 
in Table 2, and Figure 4 visualizes the mode shapes. These identification results will feed the ranking process expressed in Section 6.
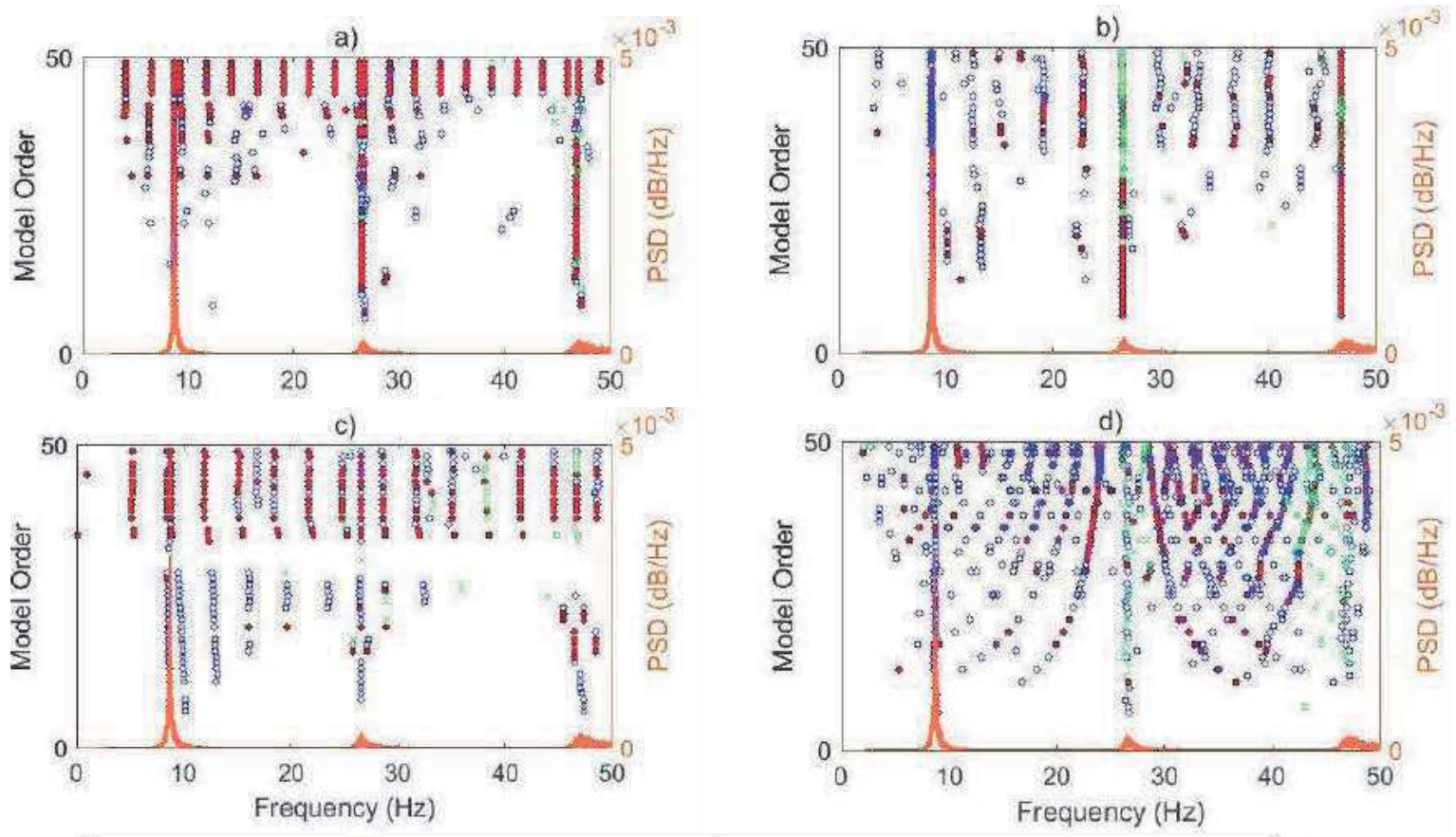

- stable pole o stable freq. \& MAC stable freq. \& damp. " stable freq.

Figure 3. Stabilization diagrams from a) OKID, b) D-SSI, c) SRIM and d) ARX, respectively.

Table 2. Identified modal frequencies and damping ratios of the numerical beam.

\begin{tabular}{lllllll}
\hline & \multicolumn{3}{c}{ Frequency $(\mathrm{Hz})$} & \multicolumn{3}{c}{ Damping ratio } \\
\cline { 2 - 7 } Method & Mode 1 & Mode 2 & Mode 3 & Mode 1 & Mode 2 & Mode 3 \\
\hline Actual & 8.679 & 26.493 & 46.786 & 0.020 & 0.020 & 0.020 \\
FDD & 8.691 & 26.465 & 46.875 & 0.017 & 0.017 & 0.017 \\
OKID & 8.672 & 26.490 & 46.777 & 0.022 & 0.020 & 0.020 \\
D-SSI & 8.681 & 26.490 & 46.782 & 0.020 & 0.020 & 0.020 \\
SRIM & 8.779 & 26.223 & 46.122 & 0.018 & 0.016 & 0.015 \\
ARX & 8.748 & 26.508 & 46.852 & 0.031 & 0.016 & 0.015 \\
\hline
\end{tabular}

According to Table 2, the lowest error percentage between actual values and individual methods are D-SSI, D-SSI, D-SSI for $1^{\text {st }}(0.027 \%), 2^{\text {nd }}(0.013 \%)$, and $3^{\text {rd }}(0.009 \%)$ modal frequencies, DSSI, D-SSI, and D-SSI, for $1^{\text {st }}(0 \%), 2^{\text {nd }}(0.5 \%)$ and $3^{\text {rd }}(2 \%)$ modal damping ratios, respectively. Regarding mode shapes, D-SSI, OKID, and OKID has the smallest 1-MAC values such as 0.00, 0.0001 , and 0.00 , respectively. It is expected that the ranking procedure described in the following section favours a similar outcome. 


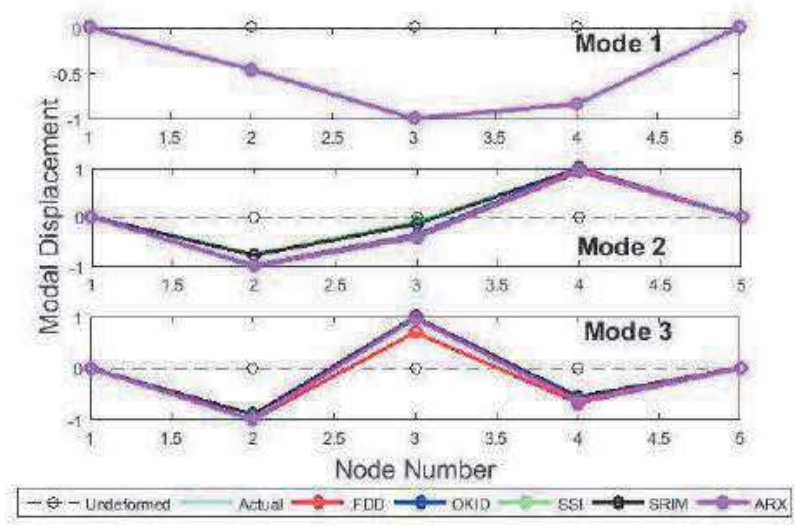

Figure 4. Identified mode shapes of the numerical beam (elevation view).

\section{Bridge 1: Shaking Table Experiments}

The system identification methods discussed in Section 2 are initially used to identify modal parameters of a quarter-scale bridge structure, Bridge 1, which is subjected to seismic shaking table tests at the University of Nevada, Reno. The details of the experimental setup, procedure, and identification results are presented in the following subsections.

\subsection{Setup and Procedure}

The first testbed presented in this study is a two-span, three-bent reinforced concrete bridge structure that is subjected to shaking table tests under the supervision of Professor Saiidi and Sanders. The bridge structure, Bridge 1, has three bents, each consisting of two columns with the same cross-sections and material properties. However, each of these bents has a different height, dominating torsional behaviour in dynamic characteristics. Additional masses are added on top of the outer bents to represent the adjacent spans of a typical bridge. The structure is excited in the transverse direction by three separate, but synchronous shaking table tests. Eleven accelerometers are placed on the shaking tables, bent columns, and bridge deck, to measure input ground motion and structural response in the excitation direction. Figure 5 shows the sensor orientation, configuration, and 3-dimensional bridge model. Extensive documentation and dataset are provided on NEES Project Warehouse, Large-scale experimental seismic studies of a two-span reinforced concrete bridge system, which is accessible online from https://www.designsafe-ci.org/data/browser/public/nees.public/NEES-2005-0032.groups/. The condensed review of the testing procedure can be found in (Johnson N. R., 2008) and a detailed report provided by NEES website (Johnson, N., 2006) as well as a large number of studies presented by (Soyoz, S., \& Feng, M. Q., 2008), (Chen, 2008), (Banerjee, 2008), (Soyoz, S., Feng, M. Q., \& Shinozuka, M., 2009), (Johnson N. S., 2009) (Johnson N. S., 2009), (Frizzarin, 2010), (Jafarkhani, 2011), (Ozer, E., \& Soyoz, S., 2015), (Banerjee, S., \& Chi, C., 2013), (Saiidi, 2013), and (Ozer, E., Feng, M. Q., \& Feng, D., 2015). 
The structure is subjected to several different shaking table tests of varying intensities. As the intensity of shaking table tests increases, the structure experiences progressive damage, which is documented by crack marks and strain gauge measurements. The shaking table tests include high-amplitude, narrow-band earthquake excitations, as well as low-amplitude, broad-band white noise excitations between each damaging event. Assuming that the structural integrity does not change during white noise excitations, the system can be considered as lineartime-invariant. Therefore, the proposed methods can be used for system identification. What is more, white noise excitation has no particular frequency content; thus, structural response under white noise excitation can be reduced to an output-only system identification problem. Hence, the data used in this study is limited to the five-channel data as output response obtained from Sensor 4, 5, 7, 8, and 11 located on the bridge deck.

Four different sets of white noise response data are used to investigate the change in structural damage throughout the shaking table tests. Between each of these sets, many earthquake tests are performed to introduce structural damage. These tests are conducted with a sampling rate of 200 $\mathrm{Hz}$ leading to a Nyquist frequency of $100 \mathrm{~Hz}$. To avoid direct current components, bias, and aliasing effects, the vibration signals are band-pass filtered with lower and upper cutoff frequencies of 0.5 and $49 \mathrm{~Hz}$, respectively. Figure 6 shows the acceleration time history and the Fourier spectra of Set 1-4 measured by Sensor 7. The peak values on each of these spectra show that the dominant peak shifts leftwards as a result of the damage progress. In other words, it can be observed that modal frequencies decrease as the shaking table tests proceed, and damage level increases.

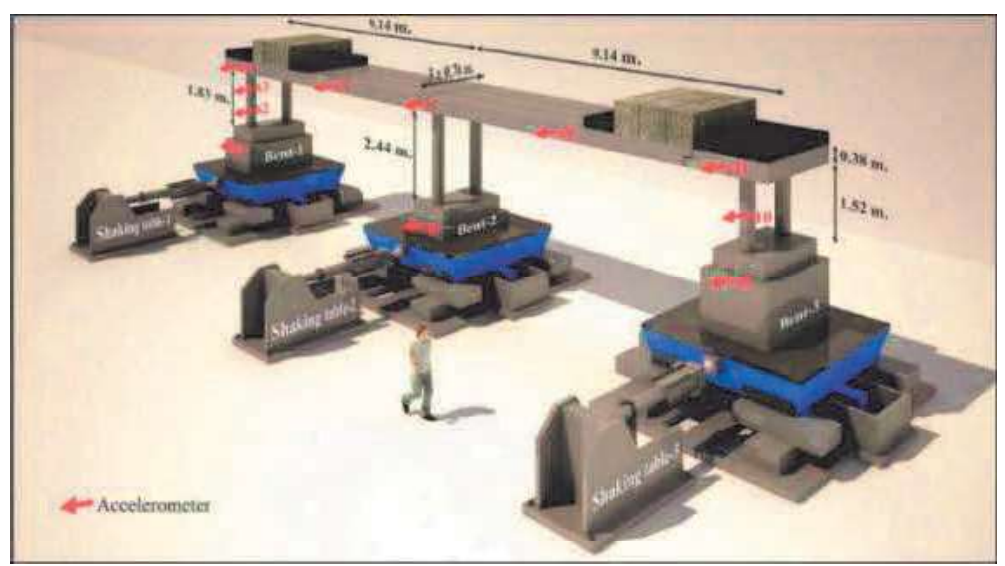

Figure 5. Experimental setup and sensor layout of the Bridge 1 test, conducted at UN, Reno. 

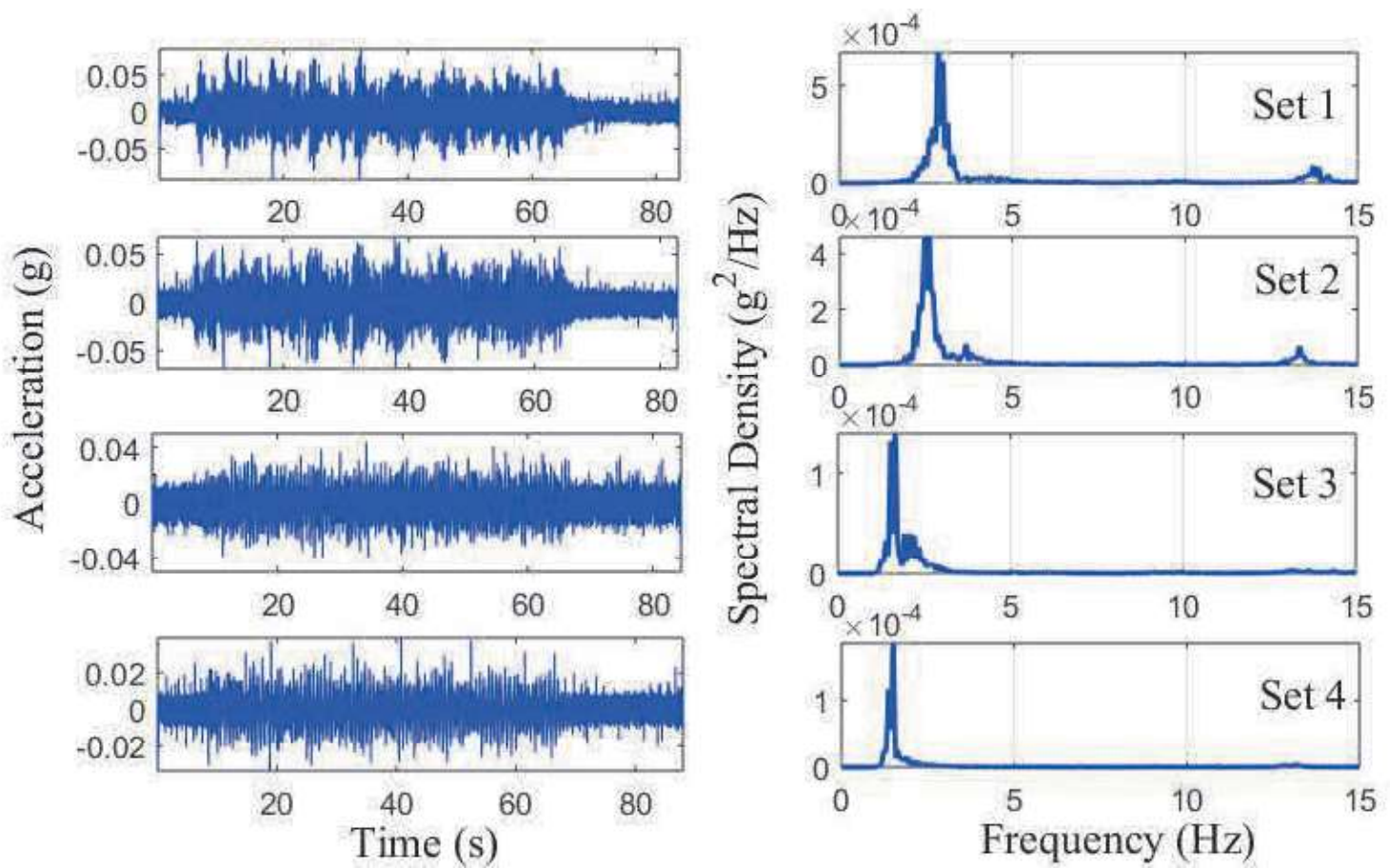

Figure 6. Acceleration time history and Fourier spectra at Sensor 7.

\subsection{Identification Results}

Using the time and the frequency domain methods discussed in Section 2, each of the data sets corresponding to a different structural state is used for system identification. The scope of the identification study only covers the $1^{\text {st }}, 2^{\text {nd }}$, and $3^{\text {rd }}$ modes of vibration in the transverse direction, which is the direction of excitation. Table 3 presents the $1^{\text {st }}, 2^{\text {nd }}$, and $3^{\text {rd }}$ identified modal frequencies and damping ratios corresponding to different data sets (Set 1, 2, 3, and 4) and identification methods (FDD, OKID, D-SSI, SRIM and ARX). Figure 7 shows mode shapes obtained from different data sets and identification methods.

Table 3. Identified modal frequencies and damping ratios of Bridge 1.

\begin{tabular}{clccccccccc}
\hline \multirow{2}{*}{ Mode No. } & \multirow{2}{*}{ Method } & \multicolumn{3}{c}{ Frequency (Hz) } & \multicolumn{5}{c}{ Damping ratio (\%) } \\
\cline { 3 - 10 } & & Set 1 & Set 2 & Set 3 & Set 4 & Set 1 & Set 2 & Set 3 & Set 4 \\
\hline \multirow{4}{*}{1} & FDD & 2.93 & 2.54 & 1.56 & 1.56 & 5.70 & 6.12 & 2.76 & 2.76 \\
& OKID & 2.96 & 2.43 & 1.42 & 1.46 & 2.73 & 3.81 & 3.32 & 2.49 \\
& D-SSI & 2.84 & 2.51 & 1.68 & 1.54 & 5.29 & 3.43 & 2.79 & 2.11 \\
& SRIM & 2.85 & 2.48 & 1.72 & 1.53 & 0.5 & 0.2 & 0.90 & 0.85 \\
& ARX & 3.03 & 2.46 & 1.64 & 1.54 & 4.76 & 5.05 & 2.99 & 2.34 \\
\hline \multirow{4}{*}{2} & FDD & 3.71 & 3.32 & 1.76 & 1.56 & 5.70 & 6.12 & 2.76 & 2.76 \\
& OKID & 3.77 & 3.69 & 2.02 & 1.71 & 3.25 & 6.34 & 3.01 & 2.54 \\
& D-SSI & 3.84 & 3.54 & 2.05 & - & 6.17 & 6.82 & 6.95 & - \\
& SRIM & 3.90 & 3.34 & 2.28 & - & 0.13 & 1.23 & 1.77 & -
\end{tabular}




\begin{tabular}{cccccccccc} 
& ARX & 3.82 & 3.66 & 1.89 & 1.60 & 4.62 & 7.46 & 5.52 & 3.76 \\
\hline \multirow{3}{*}{3} & FDD & 13.67 & 13.28 & 13.09 & 13.18 & 5.70 & 6.12 & 2.76 & 2.48 \\
& OKID & 13.77 & 13.33 & 13.35 & 13.10 & 2.86 & 1.42 & 2.08 & 1.50 \\
& D-SSI & 13.82 & 13.35 & 13.52 & 13.14 & 3.46 & 4.03 & 7.80 & 3.92 \\
& SRIM & 13.85 & 13.42 & 13.11 & 13.12 & 0.1 & 0.15 & 1.14 & 0.27 \\
& ARX & 13.98 & 13.27 & 12.94 & 13.00 & 3.84 & 1.42 & 2.30 & 1.94 \\
\hline
\end{tabular}

Note: $2^{\text {nd }}$ mode cannot be captured using D-SSI and SRIM based on Set 4 data.
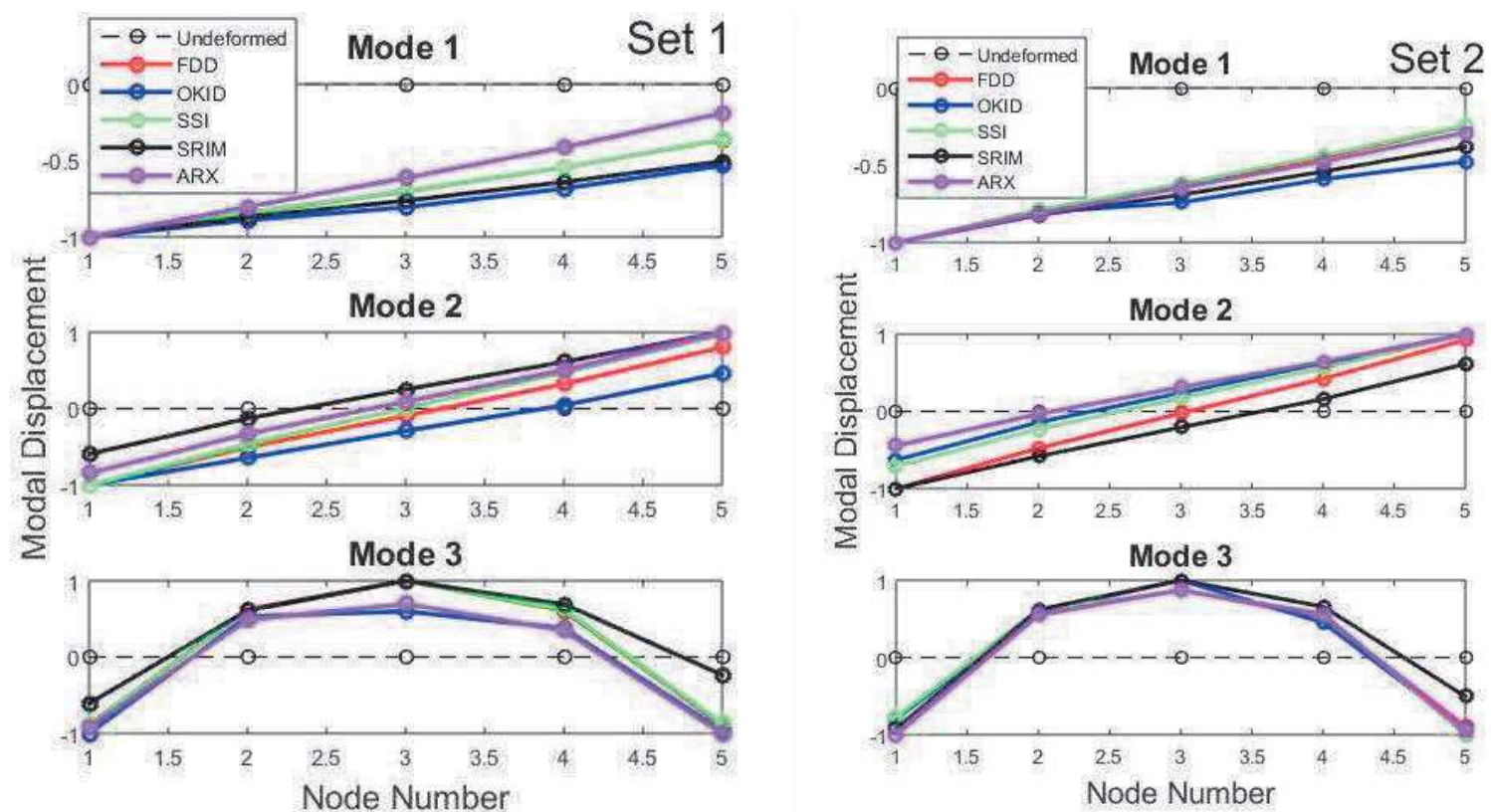
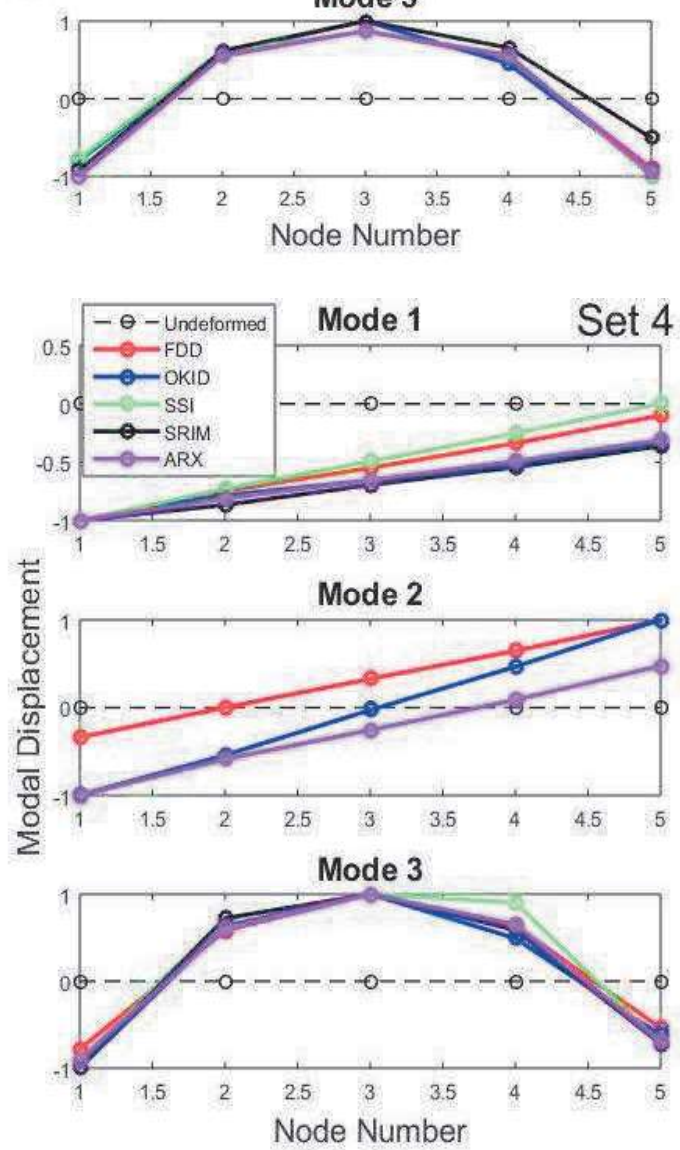

Figure 7. Identified mode shapes of Bridge 1 (top view). 


\section{Bridge 2: Field Tests}

The second testbed is a single-span steel link bridge, which serves as a connection between two multistory buildings at Columbia University Morningside Campus. The following subsections explain the details regarding the structural properties, instrumentation, and identification results, respectively.

\subsection{Structural Layout}

The bridge is instrumented with six accelerometers oriented in the vertical direction; therefore, vibration characteristics in the lateral and longitudinal direction are omitted in this study. The sensor configuration spans the entire bridge in longitudinal direction evenly spaced at six locations. Therefore, the proposed arrangement does not intend to reveal torsional modes, although peaks corresponding to these modes are observed in the frequency domain. The instrumentation photographs and bridge dimensions are shown in Figure 8.

Extensively large-sized data ( 5 hours) is obtained at nighttime to minimize pedestrian-induced vibration and take measurements under ambient vibration. The data is sampled at $100 \mathrm{~Hz}$, and band-pass filtered with cutoff frequencies of 0.5 and $49 \mathrm{~Hz}$. Figure 9 shows acceleration time histories and Fourier spectra obtained from Sensor 4, which is close to the mid-span of the bridge structure. Almost no change in vibration amplitude and stable spectral characteristics over time shows that the ambient vibration conditions are maintained throughout the tests.

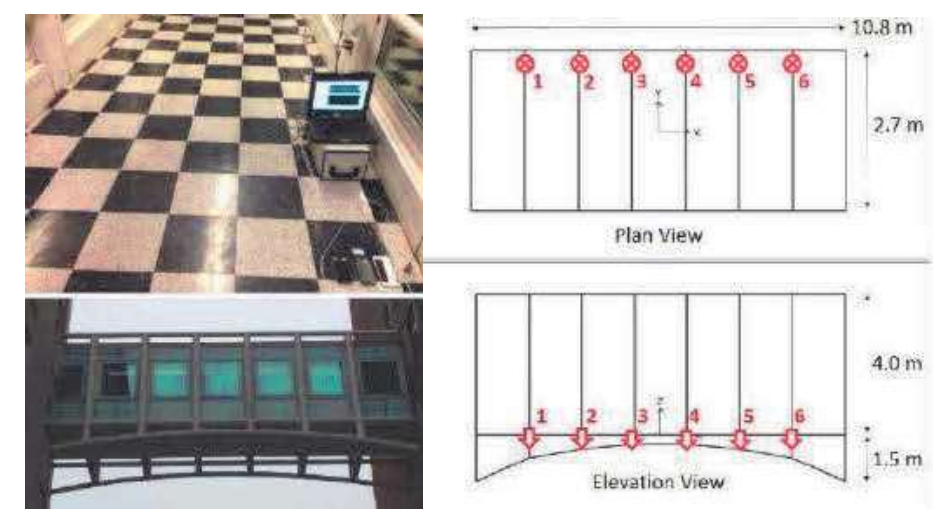

Figure 8. Inner, outer views, and sketches of Bridge 2, connecting Mudd-Schapiro Buildings. 

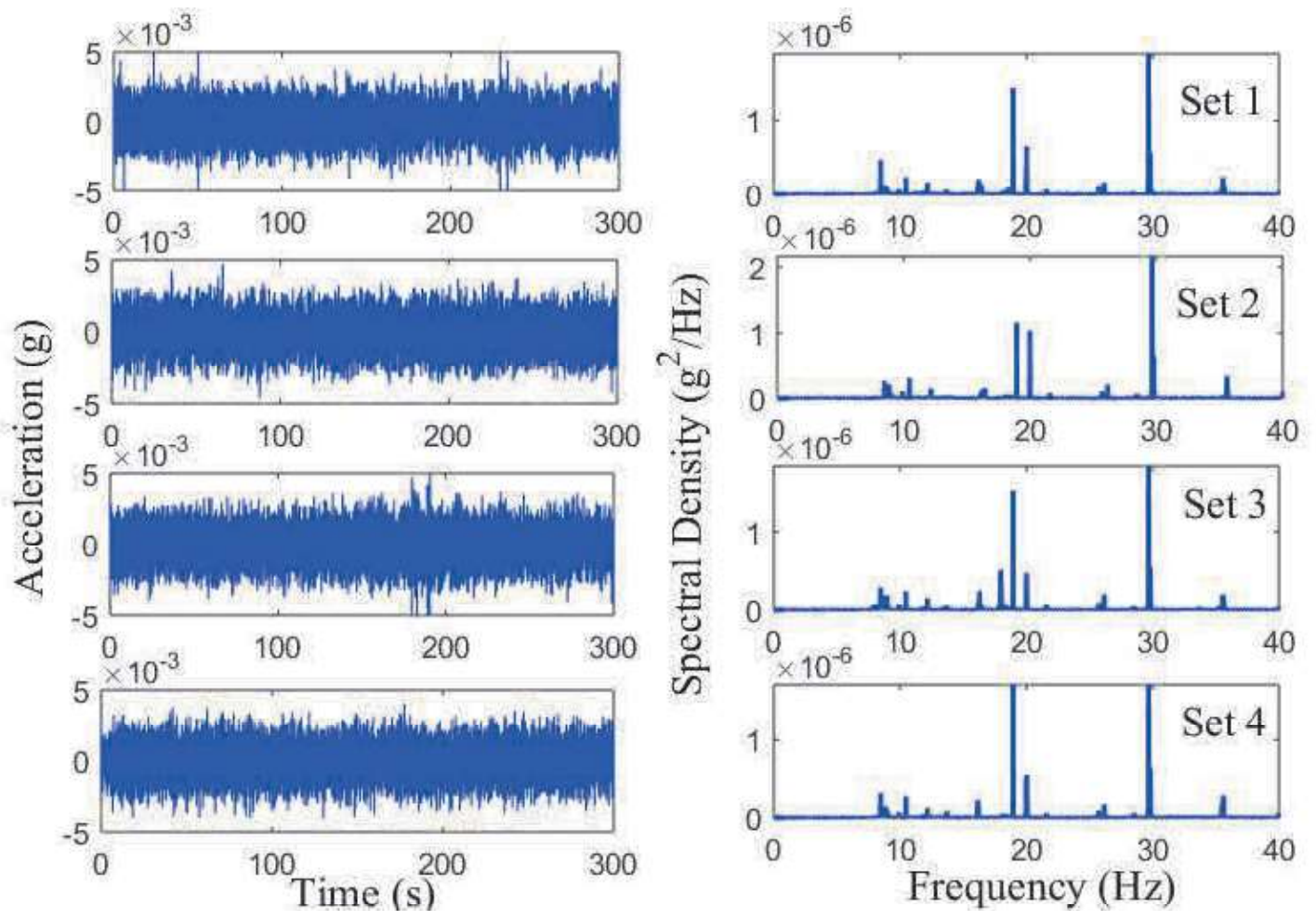

Figure 9. Acceleration time history and Fourier spectra at Sensor 4.

\subsection{Identification Results}

System identification is performed using six sensor data obtained from ambient vibration measurements. Low-amplitude ambient vibrations include a wide range of frequencies, which can be idealized as an output-only identification problem. Therefore, using a similar procedure as described before, in order to use OKID, D-SSI, SRIM, and ARX methods, input data is considered as an array of zeros. The identification results provide the $1^{\text {st }}, 2^{\text {nd }}$, and $3^{\text {rd }}$ modes of vibration in the vertical direction, which is the measurement direction of sensors. Table 4 shows the $1^{\text {st }}, 2^{\text {nd }}$, and $3^{\text {rd }}$ modal frequencies and damping ratios obtained from FDD, OKID, D-SSI, SRIM, and ARX methods. Figure 10 shows the $1^{\text {st }}, 2^{\text {nd }}$, and $3^{\text {rd }}$ mode shapes obtained from FDD, OKID, D-SSI, SRIM, and ARX methods.

Table 4. Identified Modal Frequencies and Damping Ratios of Bridge 2.

\begin{tabular}{clllllllll}
\hline \multirow{3}{*}{ Mode No. } & \multirow{2}{*}{ Method } & \multicolumn{3}{c}{ Frequency $(\mathrm{Hz})$} & \multicolumn{5}{c}{ Damping ratio (\%) } \\
\cline { 3 - 11 } & & Set 1 & Set 2 & Set 3 & Set 4 & Set 1 & Set 2 & Set 3 & Set 4 \\
\hline \multirow{3}{*}{1} & FDD & 8.79 & 8.59 & 8.79 & 8.50 & 1.75 & 1.92 & 2.09 & 1.68 \\
& OKID & 8.67 & 8.60 & 8.93 & 8.91 & 3.48 & 1.69 & 3.43 & 3.46 \\
& D-SSI & 8.60 & 8.62 & 8.47 & 8.64 & 3.82 & 3.37 & 6.20 & 3.62 \\
& SRIM & 8.72 & 8.65 & 8.99 & 8.65 & 1.76 & 2.75 & 1.84 & 0.30 \\
& ARX & 8.57 & 8.61 & 8.56 & 8.67 & 4.33 & 4.23 & 4.44 & 3.65 \\
\hline 2 & FDD & 18.95 & 18.95 & 18.95 & 18.95 & 1.75 & 1.92 & 2.09 & 1.68
\end{tabular}


- SKID $\rightarrow$ SSI $\rightarrow$ SRIM 1
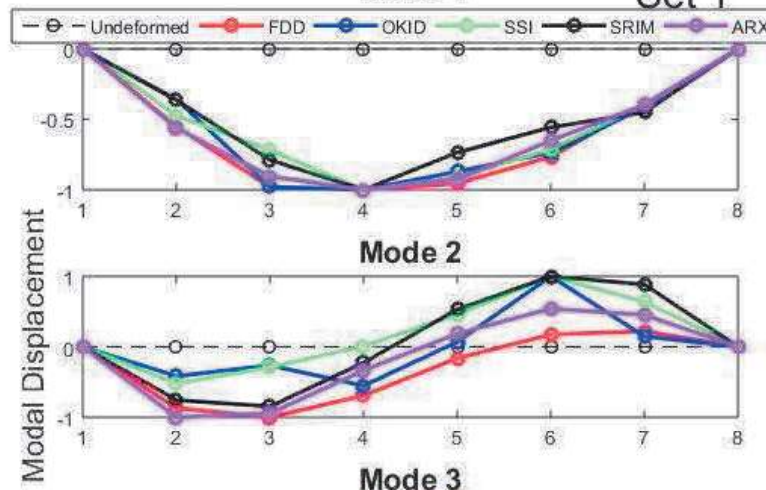

$\begin{array}{lllllllll}\text { OKID } & 18.23 & 17.98 & 18.00 & 18.24 & 2.08 & 0.40 & 1.15 & 1.56\end{array}$

$\begin{array}{lllllllll}\text { D-SSI } & 18.21 & 17.72 & 18.10 & 18.24 & 4.50 & 0.84 & 1.08 & 2.69\end{array}$

$\begin{array}{lllllllll}\text { SRIM } & 18.31 & 17.96 & 18.05 & 18.23 & 0.33 & 0.22 & 0.18 & 0.15\end{array}$

\begin{tabular}{lllllllll} 
ARX & 18.45 & 18.14 & 18.08 & 18.43 & 2.85 & 2.57 & 1.28 & 2.08 \\
\hline
\end{tabular}

\begin{tabular}{llllllllll}
3 & OKID & 29.69 & 29.71 & 29.69 & 29.70 & 0.09 & 0.05 & 0.06 & 0.08 \\
& D-SSI & 29.70 & 29.69 & 29.70 & 29.71 & 0.06 & 0.11 & 0.10 & 0.06 \\
& SRIM & 29.61 & 29.69 & 29.63 & 29.69 & 0.61 & 0.02 & 0.48 & 0.02 \\
& ARX & 29.69 & 29.65 & 29.69 & 29.70 & 0.20 & 0.91 & 0.36 & 0.28 \\
\hline
\end{tabular}




\section{Comparative and Collaborative Analysis}

In the previous sections, FDD, OKID, D-SSI, SRIM, and ARX system identification methods are applied on two exemplary bridge vibration datasets for the identification of modal parameters as damage indicators. However, looking at the individual results, one can see that the identification results from different methods do not perfectly fit each other, and there is particular dispersion in terms of modal frequencies, mode shapes, and damping ratios. Such non-uniformity of identified modal parameters due to different methods include the damaged and undamaged cases. In addition to the environmental variation of dynamic behaviour, the sensitivity of modal parameters to structural damage, and lack of input measurements and high noise in the response signals, incorrect identification results can mislead the opinions regarding the structural state. A comparison of different identification results is a starting action to overcome this problem; however, it is not enough to get the maximum outcome from the advantages of a collaborative system identification algorithm plethora. In this paper, a method-centric synergistic scheme guides the quantitative combination of FDD, OKID, D-SSI, SRIM, and ARX. The ranking procedure inheriting the optimal identification method findings is discussed as follows.

Method-centric approach lines up the multiple identification methods according to their correlation with the remainder of the techniques in which the highest rank technique is ultimately selected in terms of modal parameters. For modal frequencies and damping ratios, the difference between the two methods can impose the quantity of lack of agreement through error sums. Without a reference value, the Natural Frequency Difference (NFD) suggests to use the minimum value as the denominator (see (Ewins, 2009), (Ebrahimi, 2013), and similar comparisons can be made accordingly.

$$
\begin{gathered}
\Delta f_{j}^{i}(\%)=\left|\frac{f_{\text {method }_{i}}-f_{\text {method }_{j}}}{\min \left(f_{\text {method }_{i}}, f_{\text {method }_{j}}\right)}\right| \cdot(100) \\
\Delta \xi_{j}^{i}(\%)=\left|\frac{\xi_{\text {methodi }}-\xi_{\text {method }_{j}}}{\min \left(\xi_{\text {method }_{i}}, \xi_{\text {method }_{j}}\right)}\right| \cdot(100)
\end{gathered}
$$

where $\boldsymbol{f}$ and $\boldsymbol{\xi}$ are modal frequencies and damping ratios. Sum of errors related to the method and mode numbers will return the eventual agreement between a method and the rest of the method population.

$$
\begin{aligned}
& \operatorname{Rank} \operatorname{Term}(f)_{i^{\text {th }}{ }_{\text {Method }}}=p_{i} \sum_{j=1}^{M=5} \sum_{k=1}^{N=3} \Delta f_{j k}^{i} \\
& \operatorname{Rank} \operatorname{Term}(\xi)_{i^{\text {th }}{ }_{\text {Method }}}=p_{i} \sum_{j=1}^{M=5} \sum_{k=1}^{N=3} \Delta \xi_{j k}^{i}
\end{aligned}
$$

where $i, j$, and $k$ refer to the method of interest, method of comparison and modal parameter indices, respectively. The smaller the error, the better match between the two methods in comparison occurs. Such a comparison process can perform for each of the alternatives and mode numbers, eventually leads to the error sum for each particular technique. Moreover, $p_{i}$ 
corresponds to the penalty coefficient, which weighs prioritized methods over others if a prior opinion is present in terms of algorithm reliability. For cases where such information is absent, all methods are treated as equivalent, and the penalty coefficient becomes unity for each method by default. It is noted that self-comparison returns empty-sequence results by nature and is uninfluential in the final rank due to zero error contribution.

In addition to the modal frequencies and damping ratios, mode shapes can serve as ranking indicators quantifying coherence among different identification methods. In the case of mode shapes, such a ranking method can utilize via modal assurance criteria (MAC), quantifying the similarity between two modes. The MAC value lies within the range 0 to 1 , defining the interval from no correlation to maximum correlation. Similar to the zero addends given for modal frequency and damping ratio, self-comparison resulting in zero addends will not affect the overall rank of the method.

Modal Assurance Criteria (MAC) can be formulated as follows,

$$
M A C_{j}^{i}=\left|\frac{\left|\boldsymbol{\Phi}_{\text {method }_{i}}{ }^{T} \cdot \boldsymbol{\Phi}_{\text {method }_{j}}\right|^{2}}{\left(\boldsymbol{\Phi}_{\text {method }_{i}}{ }^{T} \cdot \boldsymbol{\Phi}_{\text {method }_{i}}\right) \cdot\left(\boldsymbol{\Phi}_{\text {method }_{j}}{ }^{T} \cdot \boldsymbol{\Phi}_{\text {method }_{j}}\right)}\right|
$$

where $\boldsymbol{\Phi}$ represents the mode shape vector. If a similar ranking procedure repeats for each of $M$ identification methods and $N$ mode shapes, an eventual value associating one mode's closeness with the others can infer the rank.

$$
\operatorname{Rank} \operatorname{Term}(\Phi)_{i^{\text {th }}{ }_{\text {Method }}}=p_{i} \sum_{j=1}^{M=5} \sum_{k=1}^{N=3}\left(1-M A C_{j k}^{i}\right)
$$

The scheme expressed above identifies the best-ranked identification method; however, it requires further information to quantify the agreement between the method population contributors. In other words, high intra-agreement likely represents a more confident identification, whereas, dispersion among the proposed methods implies more uncertainty in the synergistic identification results. Thus, quantifying this uncertainty can specify a deviation range for better representation of the collaborative finding's reliability. An additional term incorporating deviation among methods, yet, disregarding the outliers can be represented with median absolute deviation (MAD) accompanying the synergistic result. More complex ensemble learning methods covering uncertainty can also be found in the literature (Hastie, T., Tibshirani, R., \& Friedman, J., 2009) (Polikar, 2009). It should be noted that identification uncertainty within a particular method is omitted in this paper; however, it can be incorporated with probabilistic modal analysis approaches. The MAD values for each of the case studies are summarized in Table 8.

As a result of the process mentioned above, the highest frequency, damping ratio, and mode shape rank will refer to the method with minimal values. To implement the ranking scheme, FDD, OKID, D-SSI, SRIM, and ARX are quoted as Method 1 to 5. The error rates in terms of frequency (Eq. 44) and damping ratio (Eq. 45) and coherence of mode shape (Eq. 48) are visualized for each bridge testbed in Figure 11 (numerical example), Figure 12 (Bridge 1 Set 1) 
and Figure 13 (Bridge 2 Set 1). Table 5, 6, and 7 presents the summary of the ranking results (Eq. 46, 47 and 49) and the chosen identification result for all data sets from the numerical example, Bridge 1 and Bridge 2, respectively. According to Table 5, outperforming system identification methods are D-SSI, D-SSI, and OKID for modal frequencies, damping ratios, and mode shapes, respectively. The actual values from Table 2 (D-SSI for modal frequencies and damping ratios, dominantly OKID for mode shapes) agree well with these outcomes.
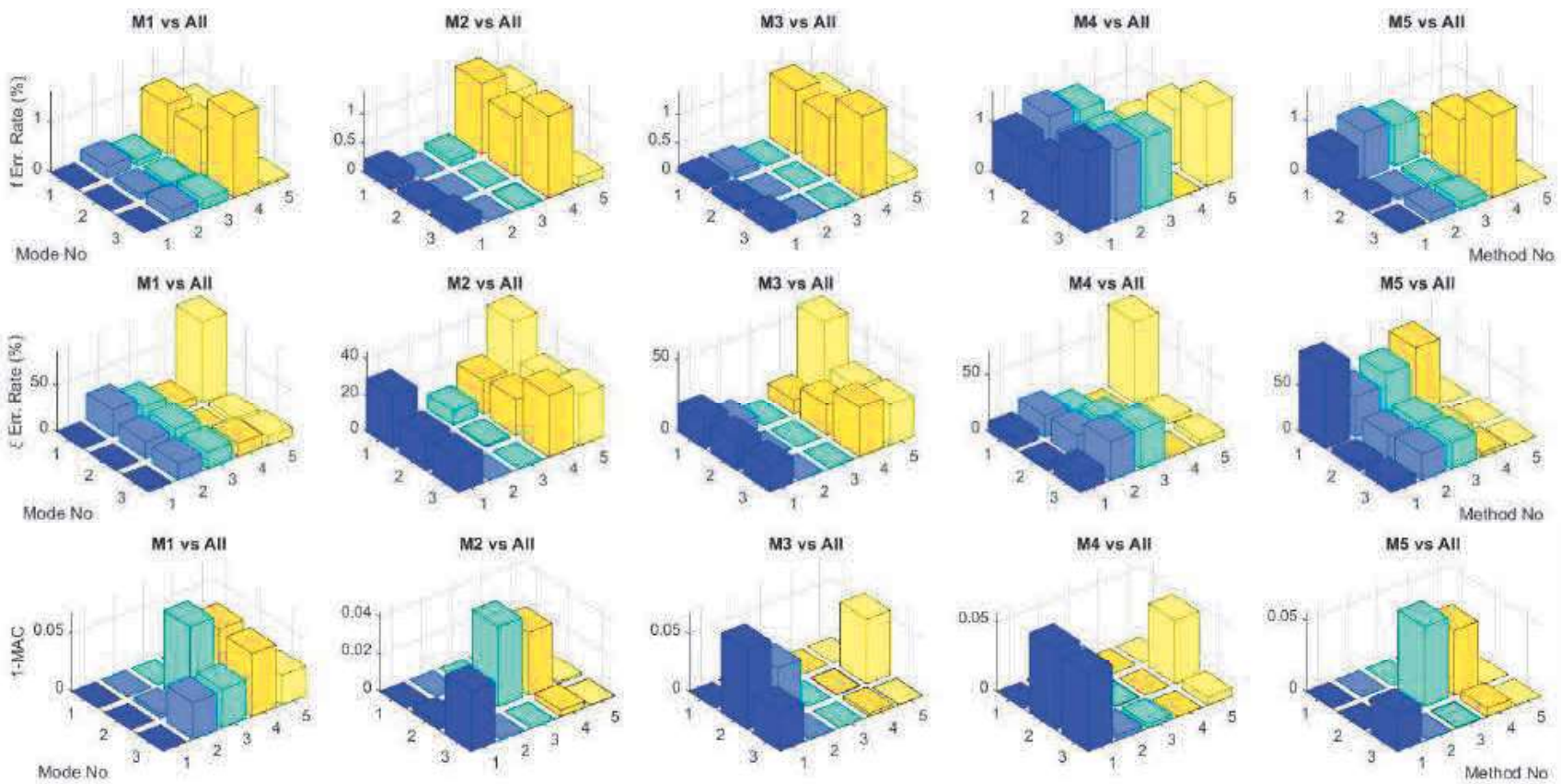

Figure 11. Modal Parameter Similarity Bar Chart (numerical example).

Table 5. Rank Chart for the numerical example (* smallest value favourable).

\begin{tabular}{cll}
\hline Parameters & Methods & Set (Only 1) \\
\hline $\boldsymbol{f}$ & FDD & 0.0536 \\
& OKID & 0.0542 \\
& D-SSI & $0.0509^{*}$ \\
& SRIM & 0.1381 \\
& ARX & 0.0598 \\
\hline $\boldsymbol{\xi}$ & FDD & 2.4783 \\
& OKID & 2.4397 \\
& D-SSI & $2.4083^{*}$ \\
& SRIM & 2.4374 \\
& ARX & 3.8898 \\
\hline $\boldsymbol{\Phi}$ & FDD & 0.2754 \\
& OKID & $0.1156^{*}$ \\
& D-SSI & 0.2047 \\
& SRIM & 0.2040 \\
& ARX & 0.1355 \\
\hline
\end{tabular}



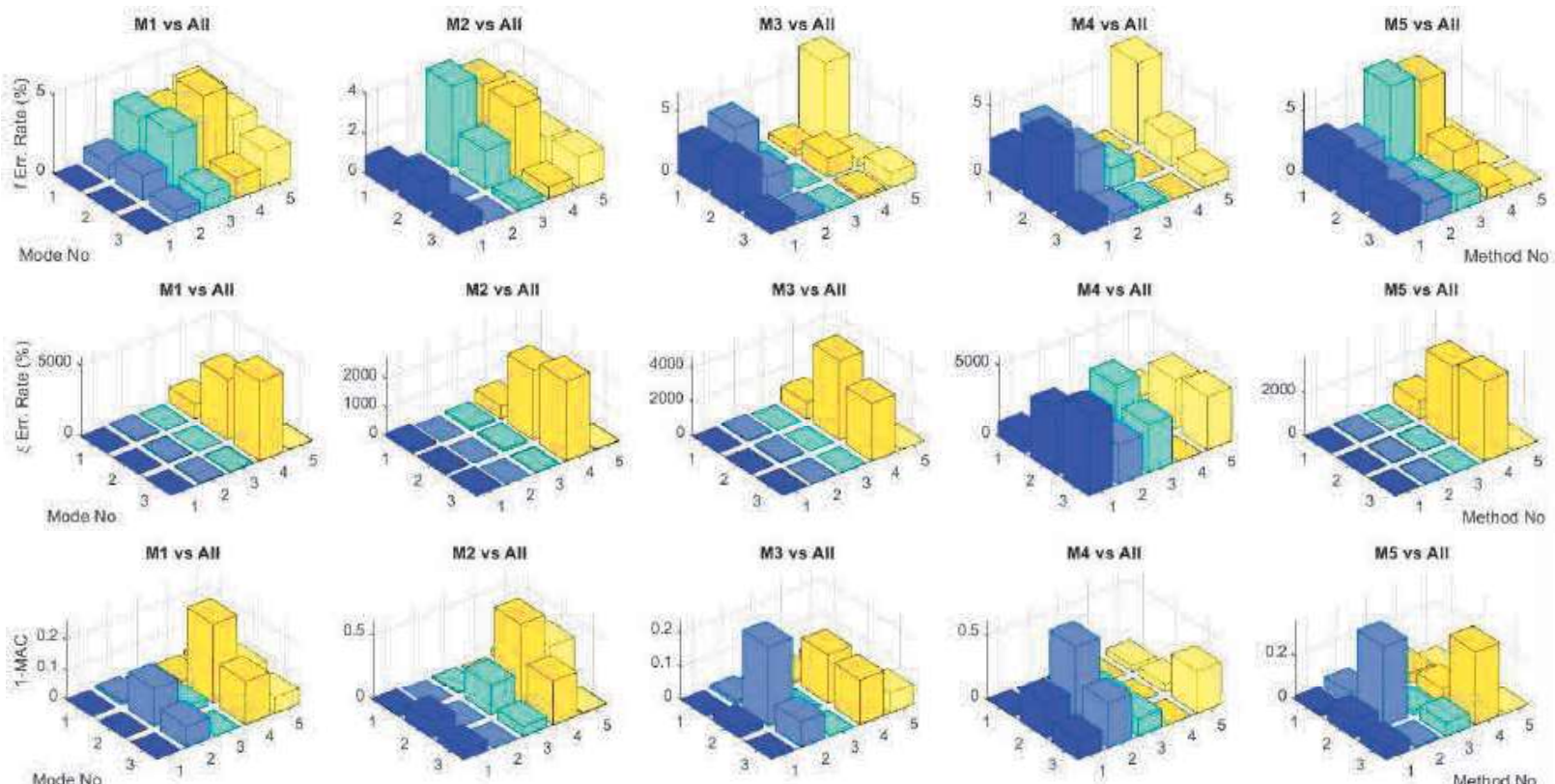

Figure 12. Exemplary Modal Parameter Similarity Bar Chart (Bridge 1, Set 1).
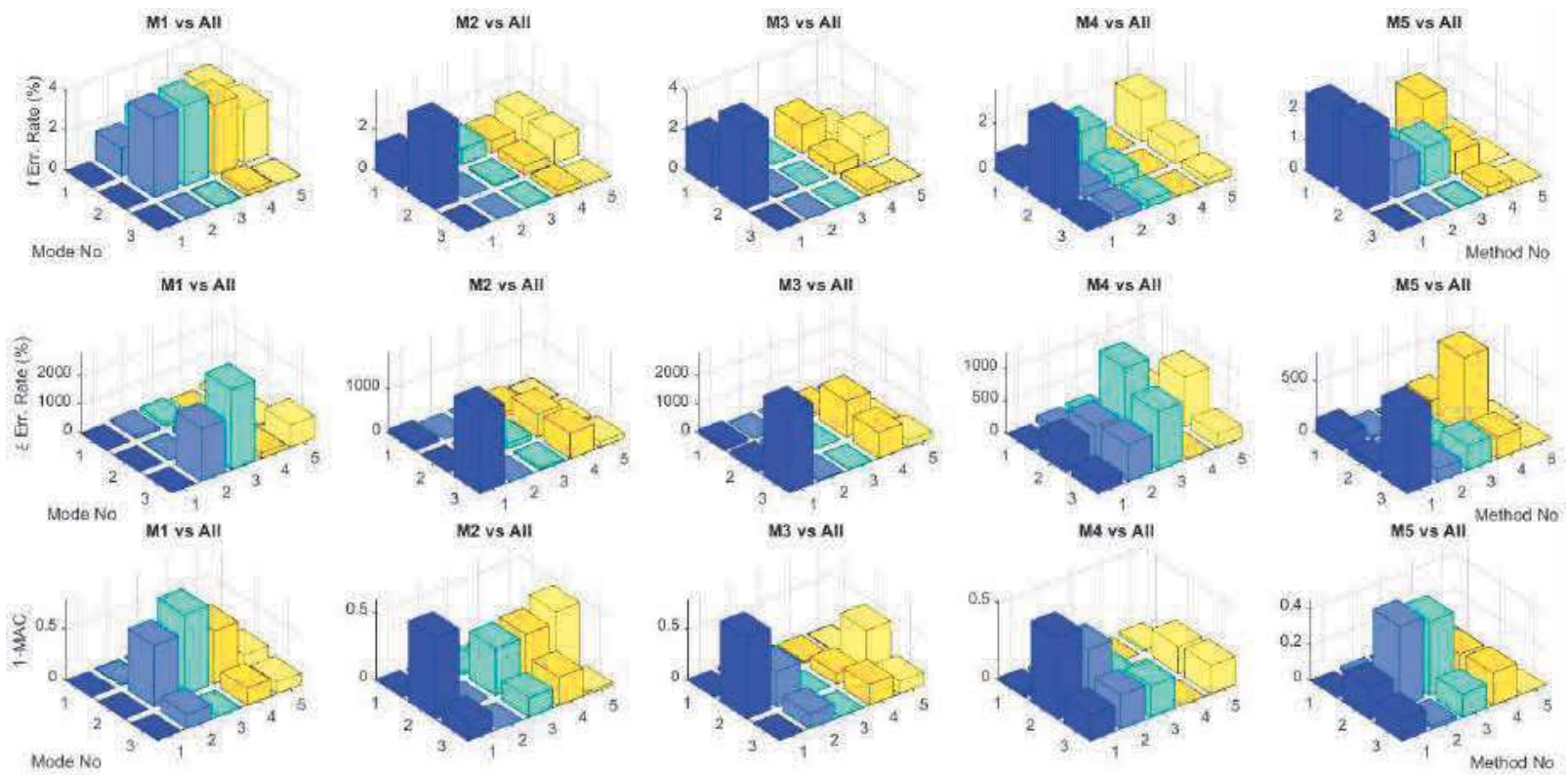

Figure 13. Exemplary Modal Parameter Similarity Bar Chart (Bridge 2, Set 1).

Table 6. Rank Chart for Bridge 1 (* smallest value favourable).

\begin{tabular}{clllll}
\hline Parameters & Methods & Set 1 & Set 2 & Set 3 & Set 4 \\
\hline $\boldsymbol{f}$ & FDD & 0.2865 & 0.4181 & 1.0824 & 0.1422 \\
& OKID & $0.2262^{*}$ & 0.3843 & 1.0476 & $0.1404^{*}$ \\
& D-SSI & 0.2471 & $0.2909^{*}$ & 0.7916 & 0.1573 \\
& SRIM & 0.2814 & 0.3572 & 1.2165 & 0.1495 \\
& ARX & 0.3121 & 0.3273 & $0.7624^{*}$ & 0.1436 \\
\hline
\end{tabular}




\begin{tabular}{llllll}
\hline$\xi$ & FDD & 113.8039 & 82.4857 & $9.2269^{*}$ & 12.6344 \\
& OKID & $62.4485^{*}$ & $37.1499^{*}$ & 10.2135 & $9.6128^{*}$ \\
& D-SSI & 93.0515 & 52.4073 & 21.0515 & 19.8320 \\
& SRIM & 335.4062 & 188.3898 & 24.5162 & 40.2845 \\
& ARX & 83.4385 & 44.4287 & 10.2999 & 12.7729 \\
\hline $\boldsymbol{\Phi}$ & FDD & 0.7920 & 0.7771 & 3.7276 & $1.1720^{*}$ \\
& OKID & 1.9102 & 0.8361 & 2.5239 & 1.1922 \\
& D-SSI & $0.7298^{*}$ & $0.6888^{*}$ & 2.5641 & 1.3399 \\
& SRIM & 2.1088 & 1.7728 & $2.2010^{*}$ & 1.1850 \\
& ARX & 1.0999 & 1.1206 & 4.7966 & 4.0954 \\
\hline
\end{tabular}

Table 7. Rank Chart for Bridge 2 (* smallest value favourable).

\begin{tabular}{clllll}
\hline Parameters & Methods & Set 1 & Set 2 & Set 3 & Set 4 \\
\hline $\boldsymbol{f}$ & FDD & 0.2138 & 0.2362 & 0.3026 & 0.2498 \\
& OKID & $0.0998^{*}$ & $0.0940^{*}$ & $0.1876^{*}$ & 0.1877 \\
& D-SSI & 0.1110 & 0.1345 & 0.2239 & 0.1045 \\
& SRIM & 0.1096 & 0.1035 & 0.2063 & $0.1023^{*}$ \\
& ARX & 0.1226 & 0.1025 & 0.1885 & 0.1142 \\
\hline$\xi$ & FDD & 66.5730 & 165.6427 & 78.7575 & 154.1031 \\
& OKID & 35.2775 & 71.7035 & 55.4915 & $48.0620^{*}$ \\
& D-SSI & 58.7014 & $38.9206^{*}$ & 38.6919 & 63.8281 \\
& SRIM & 52.3558 & 169.3686 & 46.3812 & 187.7667 \\
& ARX & $25.8819^{*}$ & 92.0870 & $23.0093^{*}$ & 50.3005 \\
\hline $\boldsymbol{\Phi}$ & FDD & 2.5769 & 2.7437 & 3.3921 & 2.8834 \\
& OKID & 2.3747 & 2.1596 & 1.9953 & 3.5139 \\
& D-SSI & 2.2246 & 1.6231 & 1.5608 & $1.6592^{*}$ \\
& SRIM & 2.0358 & 1.2405 & 2.8580 & 1.7300 \\
& ARX & $1.7307^{*}$ & $1.0923^{*}$ & $1.3238^{*}$ & 1.8534 \\
\hline
\end{tabular}

Table 8. Median absolute deviations for frequencies and damping ratios from the three case studies.

\begin{tabular}{lccccccc}
\hline Parameters & Set & $\boldsymbol{f}_{\mathbf{1}}$ & $\boldsymbol{f}_{\mathbf{2}}$ & $\boldsymbol{f}_{\mathbf{3}}$ & $\boldsymbol{\xi}_{\mathbf{1}}$ & $\boldsymbol{\xi}_{\mathbf{2}}$ & $\boldsymbol{\xi}_{\mathbf{3}}$ \\
\hline Numerical & - & 0.0194 & 0.0181 & 0.0705 & 0.0018 & 0.0009 & 0.0021 \\
\hline Bridge 1 & 1 & 0.0749 & 0.0532 & 0.0496 & 0.0094 & 0.0137 & 0.0060 \\
& 2 & 0.0299 & 0.1493 & 0.0517 & 0.0124 & 0.0048 & 0.0127 \\
& 3 & 0.0630 & 0.1251 & 0.2003 & 0.0008 & 0.0124 & 0.0039 \\
& 4 & 0.0244 & 0.0401 & 0.0245 & 0.0037 & 0.0011 & 0.0085 \\
\hline Bridge 2 & 1 & 0.0677 & 0.0976 & 0.0054 & 0.0085 & 0.0077 & 0.0014 \\
& 2 & 0.0137 & 0.1584 & 0.0054 & 0.0083 & 0.0062 & 0.0009 \\
& 3 & 0.1995 & 0.0341 & 0.0058 & 0.0134 & 0.0013 & 0.0026 \\
& 4 & 0.0200 & 0.0023 & 0.0051 & 0.0019 & 0.0040 & 0.0006 \\
\hline
\end{tabular}

\section{Results and Discussion}


Following implementation of the proposed ranking scheme and successful verification on the numerical model, this section discusses benchmark comparisons, synergistic outcomes, environmental effects on modal analysis results and frequency-damage dependence for Bridge 1 and Bridge 2. Prior studies related to Bridge 1 presented in Table 9 serve as benchmarks and support the modal identification discussions in this section.

Table 9. Identification results of Bridge 1 published by different authors.

\begin{tabular}{|c|c|c|c|c|c|c|c|c|}
\hline \multirow{2}{*}{ Authors } & \multicolumn{4}{|c|}{$1^{\text {st }}, 2^{\text {nd }}, 3^{\text {rd }}$ Frequencies $(\mathrm{Hz})$} & \multicolumn{4}{|c|}{$\mathbf{1}^{\text {st }}, 2^{\text {nd }}, 3^{\text {rd }}$ Damping Ratios ( $\left.\%\right)$} \\
\hline & Set 1 & Set 2 & Set 3 & Set 4 & Set 1 & Set 2 & Set 3 & Set 4 \\
\hline \multirow[t]{3}{*}{ Soyoz S., \& Feng, M. Q., 2008} & 2.93 & 2.54 & 1.76 & 1.56 & - & - & - & - \\
\hline & 3.70 & - & - & - & - & - & - & - \\
\hline & 13.70 & - & - & - & - & - & - & - \\
\hline \multirow[t]{3}{*}{ Frizzarin M. et al. 2010} & 2.91 & 2.53 & 1.63 & 1.51 & 7.4 & 6.6 & 2.8 & 1.3 \\
\hline & - & - & - & - & - & - & - & - \\
\hline & - & - & - & - & - & - & - & - \\
\hline \multirow{3}{*}{ Jafarkhani R., \& Masri S. F., 2011} & 3.09 & 2.46 & 1.53 & 1.39 & - & - & - & - \\
\hline & 4.15 & 3.44 & 1.82 & 1.57 & - & - & - & - \\
\hline & 12.90 & 12.33 & 11.95 & 11.94 & - & - & - & - \\
\hline \multirow[t]{3}{*}{ Ozer E., \& Soyoz, S., 2015} & 2.93 & - & - & - & 5.0 & 6.0 & 9.6 & 11.5 \\
\hline & 3.66 & - & - & - & - & - & - & - \\
\hline & 13.87 & - & - & - & - & - & - & - \\
\hline
\end{tabular}

\subsection{Bridge 1 Benchmark Comparisons}

The $1^{\text {st }}$ modal frequency results from FDD, OKID, D-SSI, SRIM, and ARX (2.93, 2.96, 2.84, 2.85, and $3.03 \mathrm{~Hz}$ respectively) corresponding to Set 1 data are compared with previous studies presented in Table 9. For instance, the errors between FDD, OKID, D-SSI, SRIM, ARX and (Soyoz, S., \& Feng, M. Q., 2008) are $\% \%, 1.0 \%, 3.1 \%, 2.7 \%$, and 3.4\%, respectively. Another example is the comparison of $2^{\text {nd }}$ modal frequencies of Set $1(3.71,3.77,3.84,3.90$, and $3.82 \mathrm{~Hz})$ with (Jafarkhani, 2011). The errors corresponding to FDD, OKID, D-SSI, SRIM, and ARX are $10.6 \%, 9.2 \%, 7.5 \%, 6.0 \%$, and $8.0 \%$ respectively. As a result, the identification frequencies slightly vary according to the identification method.

In the case of damping, the identification results are much more erroneous. For instance, FDD, OKID, D-SSI, SRIM, and ARX 1st mode damping values from Set 1 (5.7\%, 2.7\%, 5.3\%, 0.5\%, and $4.8 \%$ ) are much different from those obtained by (Frizzarin, 2010) and (Ozer, E., \& Soyoz, S., 2015). The FDD, OKID, D-SSI, SRIM, and ARX results have errors of $23 \%, 63 \%, 29 \%$, 93\%, 36\% according to (Frizzarin, 2010) and 14\%, 45\%, 6\%, 90\%, 5\% according to (Ozer, E., \& Soyoz, S., 2015). Similar substantial differences are present looking at the other sets such as Set 2 , the 1 st mode FDD, OKID, D-SSI, SRIM, ARX damping ratios with 7\%, 42\%, 48\%, 97\%, $24 \%$ errors according to (Frizzarin, 2010) and 2\%, 37\%, 43\%, 97\%, 16\% errors according to (Ozer, E., \& Soyoz, S., 2015). A similar variation pattern can be observed, looking at different data sets and different modes.

By tracking the evolution of the first, second, and third frequencies in Table 3, one can observe a gradual decrement in modal frequencies from Set 1 to 4 as well as (Soyoz, S., \& Feng, M. Q., 2008), (Frizzarin, 2010), (Jafarkhani, 2011), and (Ozer, E., \& Soyoz, S., 2015) findings. 
Regarding damping, there is a clear indication of increasing damping as damage progresses throughout the tests according to (Ozer, E., \& Soyoz, S., 2015); however, such behaviour reverses in (Frizzarin, 2010). No distinct patterns are observed looking at damping identification results of Table 3 as shaking table tests proceed from Set 1 to Set 4 . These deviations highlight the difficulties and low confidence levels in the identification of civil infrastructure damping features. Consequently, uncertainty in damping estimation is considerably higher than the one in modal frequencies; therefore, the damping ratio is less trivial as a damage quantification measure based on the results.

\subsection{Bridge 2 Benchmark Comparisons}

Similar to Bridge 1, Bridge 2 hosted a variety of modal identification studies addressing mobile, smart, and citizen-engaged identification scenarios in the last five years (Ozer, E., Feng, M. Q., \& Feng, D., 2015), (Ozer, E., \& Feng, M. Q., 2016), (Ozer, E., \& Feng, M. Q., 2017), (Ozer, E., \& Feng, M. Q., 2017), (Ozer, E., \& Feng, M. Q., 2019). In these studies, modal frequency values point out approximately $8.5,19,30 \mathrm{~Hz}$ for $1 \mathrm{st}$, 2nd, and 3rd modes, respectively. Based on Table 4 , one can see the similar values observed over different sets without any distinct change among the sets. No significant frequency difference except small deviations is perceived as the tests proceed from Set 1 to 4 since the bridge is not subject to any changes within this time interval. However, the damping ratio identification results are not as consistent, when different sets are compared with each other. This is in line with the erroneous behaviour depicted in Bridge 1, where tracking structural damage is not viable through the trend in damping ratio values, or the damping ratios are not stationary despite no structural changes.

\subsection{Comparison and Collaboration of Multiple Identification Methods}

Regarding the ranking scheme, one can see that the method with the smallest error sum is chosen for frequency, damping ratio, and mode shape identification as presented in Table 6 and 7 . As a result, the method ranking in the synergistic scheme becomes advantageous to remove specific algorithm findings which are highly uncorrelated with the others. Such rank cannot express the exact identification result, but the one which is most comprehensively confirmed by a population of different methods.

To understand how a synergistic process can merge different identification results, Figure 14 shows the fundamental frequencies of the damaged and undamaged bridges obtained from consecutive datasets. As expected, there is a $48 \%$ decline with the increased severity of earthquakes imposed on the structure when Bridge 1 taken into consideration. Such change and damage features can be more comprehensively expressed in terms of modal parameters through a model updating approach (Mottershead, 1993). In contrast, the frequency characteristics remain the same as the tests proceed in case of Bridge 2. Nevertheless, there are still minor fluctuations in Bridge 2, imposing the effects of non-damage-related features. The synergistic combination of the identification methods reduces the first modal frequency's variability from $0.52 \mathrm{~Hz}$ range to $0.33 \mathrm{~Hz}$ range (36.5\% reduction) due to the inherent outlier removal nature. For the second and third modal frequencies, the variability reduces by $79.7 \%$ and $80.0 \%$, respectively. Such fluctuation decrease can propose more robust identification performance for more extreme 
sensing scenarios, including novel SHM tools such as citizen science especially if the applications require real-time action.

It should be noted that the disagreement between methods are also quantified with MAD to express the confidence level in the optimal findings. Gray vertical lines represent the deviation from the best ranking method's identification result. Larger MAD values correspond to higher disagreement between methods. In most cases, the deviations show a range smaller than the population of results including outliers.
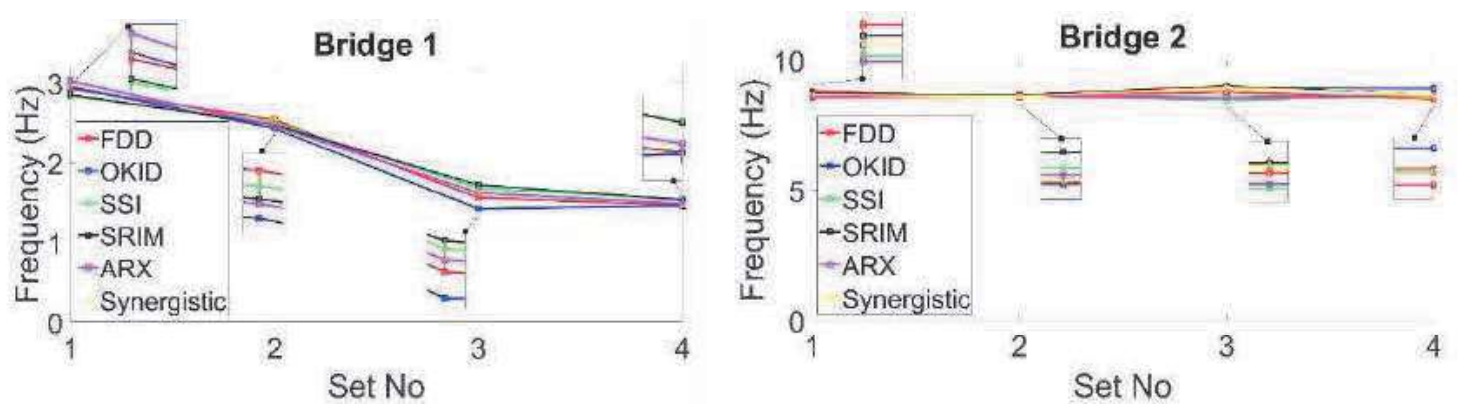

Figure 14. Progress of the fundamental frequency from different approaches and synergistic solution for the benchmark bridge testbeds.

\subsection{Consensus Difference Between Method-Centric and Parameter-Centric Approaches}

The scheme proposed in this paper can be apprehended as method-centric, that is to say, depends on identifying the outranking method rather than the statistical combination of the modal parameter findings of different algorithms. A parameter-centric form, e.g., median values, can combine system identification results per parameter from multiple methods in a similar way, and for comparison, is presented in this subsection. Table 10 summarizes the identified modal frequencies for all case studies, including the method-centric approach proposed in this paper and the median values of the parameter-centric approach. The summary includes one numerical, four sets of gradually damaged (Bridge 1), and four sets of undamaged (Bridge 2) modal frequency and damping ratio identification results. Out of the 54 comparative cases, 16 cases correspond to the same result determining the outranking modal parameter. In other words, the method-centric ranking method performs differently than a traditional averaging procedure in 38 cases. The fundamental reason behind this is that the parameter-centric approach treats each mode separately, whereas the method-centric approach perceives multiple modes together. 


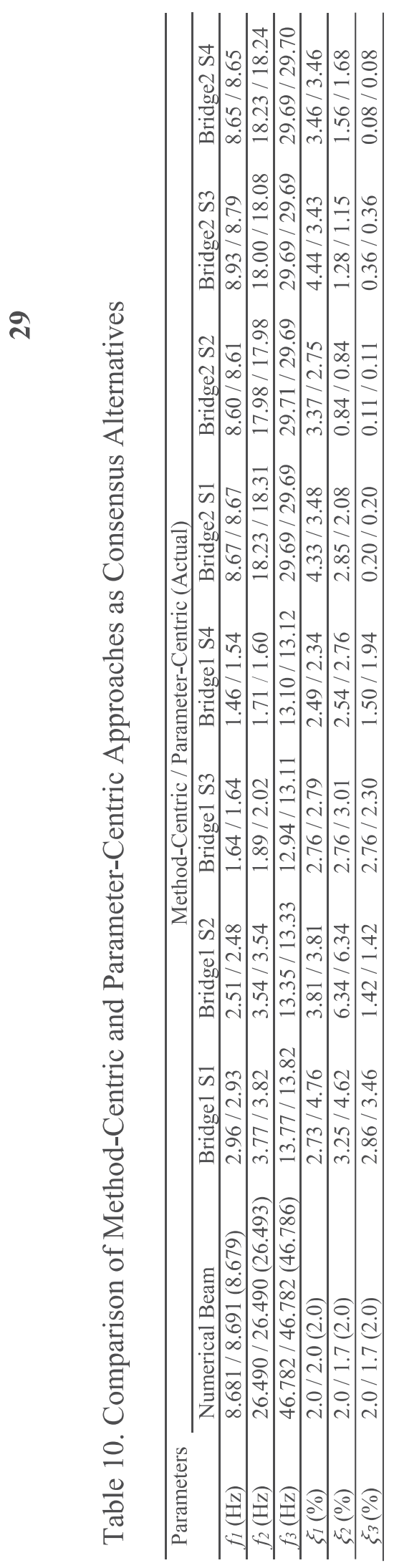
অ 
Regarding the ground truth comparisons, Bridge 1 and Bridge 2 datasets are experimental and cannot provide reference values. However, the numerical example shows that method-centric approach selects 6 values closest to the actual value out of 6 cases. This successful selection number reduces to 3 out of 6 (second and third modal frequencies and first modal damping ratio) in the parameter-centric approach based on median values. Although this is a limited observation showing the difference between the two approaches, it provides a snapshot of the method-centric ranking methodology's worthwhile performance. In addition to these results, for the proper application of the parametercentric form in real-life implementations, the authors foresee the necessity to follow additional steps securing that each identification result corresponds to the realization of interest (cleansing, clustering, and other techniques to ensure that $\mathrm{i}^{\text {th }}$ mode matches other $\mathrm{i}^{\text {th }}$ modes in the method pool). Besides, vectorial forms (i.e., mode shapes) may need additional effort to bring them onto an averaging perspective.

\subsection{Discussion on a Unified Ranking Description and Pathways for Determining Penalty Weights}

Given the existing ranking scheme, one can observe that one method can overperform others in one parameter, whereas, another method can lead to a different parameter. The proposed ranking scheme currently evaluates modal frequency, damping ratio, and mode shape separately; however, it is possible to merge multiple modal parameters in a unified domain with an integrated function. In this case, the ranking term needs to express other coefficients defining each parameter's error contribution since they are formed on a different basis. Hypothetically, such a function could take the form such as

$$
\operatorname{Rank}_{\text {Term }_{i} \text { th } M e t h o d}=p_{i} \sum_{j=1}^{M=5} \sum_{k=1}^{N=3}\left(h_{f} \Delta f_{j k}^{i}+h_{\xi} \Delta \xi_{j k}^{i}+h_{\emptyset}\left(1-M A C_{j k}^{i}\right)\right)
$$

Where $h_{f}, h_{\xi}$, and $h_{\varnothing}$ represent additional weights (modal frequency, damping ratio, mode shape) associated with the modal parameter type. The fundamental reason for these coefficients is that reliability on each parameter is likely to be different (for example, damping identification is more susceptible to error, so should be accompanied by a smaller weight).

Other than weights assigned each modal parameter, each system identification method can also involve a different level of uncertainty. As mentioned before, penalty coefficients related to each method can prioritize some methods over others based on quantified variances. In that sense, low variance terms associated with a method indicate low penalties in the method rank calculation. For example, some methods, such as time domain SSI-cov algorithms, express variance within their formulation (Mellinger, 2016) (Reynders E. M., 2016) or those in the frequency domain with Bayesian approaches (Au, 2013). For those which lack such features, additional simulation techniques may be utilized to reproduce the modal analysis findings in a randomized manner.

Alternatively, a training process can also quantify the optimal penalty and weighing coefficients with many realizations from a physical model and its probabilistic products. As can be seen, these steps can further improve the ranking scheme; on the other hand, may require additional items such as modeling information batches, high numbers of forward (response simulations) and backward (system identification) numerical analyses, and an optimization process. These incremental steps can offer a more robust identification process and potentially less bias through a systematic modification of the penalties and weights. At this stage, this paper only provides a skeleton for the synergistic modal analyses open to the aforementioned improvements.

\subsection{Discussion Concerning Environmental Effects vs Damage on Modal Characteristics}

Regarding environmental effects such as temperature on modal frequency, several studies (Figueiredo, 2011), (Dervilis, 2015), (Ubertini F. C., 2017) indicate that identified modal frequency results are 
sensitive to daily and seasonal temperature changes. Therefore, damage indication based on merely modal frequency change can lead to wrong results without consideration of variability due to environmental effects. In this study, identified modal frequency variance using laboratory measurements of Bridge 1 have ignorable environment effects due to the experimental setup condition. Other than that, natural frequency reduction throughout the shaking table tests is around $47 \%$, which would be even further amplified when stiffness degradation is of concern. For Bridge 2, unchanging modal frequency results via time using consecutive ambient measurements is due to the short duration of the entire set. The observed frequency fluctuations are within $6 \%$, which seem reasonable considering low-amplitude and high-noise vibration features. Long-term measurements could reflect variation in modal parameters due to daily and seasonal features which are expected to influence modal frequency in conjunction with damage indication. For further reference, there are examples considering temperature influence to induce changes directly related to deterioration (Soyoz, S., \& Feng, M. Q., 2009).

\subsection{Wrap-up and Additional Remarks}

In summary, in realistic modal identification cases for large-scale or full-scale structures combined using low signal to noise ratio data, reliability of identification results; therefore, quantified damage has severe handicaps without further verification/validation sources. Counteracting similar fidelity problems is viable due to the existing maturity in system identification literature and advanced distributed computational skills in modern computers, multiple techniques can deploy identification in a collaborative, yet, instantaneous manner. Moreover, a ranking procedure associating different method findings with each other and favouring the highly correlated terms is expected to reduce method-centric identification errors.

Other than these, the selection of the method population is naturally of importance. For the cases where combined method perspectives are somewhat duplicates (for example, the combination of OKID with ERA vs ERA Using Data Correlations (ERA-DC) (Juang J. N., 1988), Deterministic Intersection (DI), Deterministic Projection (DP)) (Van Overschee P., 1996), weighing functions can justifiably merge the algorithms of the same family. Similar weighing issues might also address contributions from the frequency and the time domain identifications in a more unified way. Finally, as an alternative to the method-centric unification scheme, parameter-centric schemes can also be developed, where synergistic combination uses the statistical dispersion of parameters and removal of outliers in a parameter-oriented framework (e.g., trimmed mean for modal frequencies and damping ratios, quadratic combination for mode shapes).

\section{Conclusions}

In this study, system identification and modal parameter results of two bridge structures, Bridge 1 and Bridge 2, and a numerical example are presented with an eventual collocation deployment. The numerical example is a multi-degree-of-freedom beam subjected to white noise excitations. Bridge 1 is subjected to earthquake excitations induced by multi-shaking table tests in the University of Nevada, Reno, which results in progressive damage. Between earthquake excitations, low-amplitude white noise excitation responses in the transverse direction are utilized for system identification. Bridge 2 is a pedestrian link bridge in Columbia University Morningside Campus connecting two multistory buildings, and six accelerometers in vertical direction record sequential ambient vibration measurements. One frequency domain and four time-domain identification methods are used, which are FDD, OKID-ERA, D-SSI, SRIM, and ARX, respectively. Eventually, a synergistic approach is incorporated to collectively use different identification methods in a quantitative and automated 
ranking scheme with a disagreement measure formulated via MAD. In that sense, the paper brings a collaborative solution employing numerous different algorithms which has rare examples in the literature.

The numerical beam example provide baseline values to verify the proposed ranking scheme. The ranking results from these simulations show that D-SSI outperforms for modal frequencies and damping ratios, whereas OKID is the leading method for mode shapes. The baseline modal parameters confirm these exercises. In the case of Bridge 1, system identification is conducted for four different damage states; whereas, no damage is present on Bridge 2 throughout four cascaded datasets. The results obtained from different methods show that there is a good agreement between identified modal frequencies among methods, yet, outliers exist. Regarding mode shapes, the correlation between different methods changes according to the various states; however, it reaches values above 0.9 in most cases after the synergistic combination. In contrast, identified damping ratios significantly differ according to the identification method and the datasets, which is in line with the other references studying the same testbeds. In other words, the results show that identification performance of damping ratios are more dispersed and less reliable in contrast with the modal frequencies.

Despite the variations between FDD, OKID, D-SSI, SRIM, and ARX results, it is manifest that each method is capable of tracing Bridge 1's structural damage throughout the shaking table tests with the help of decrement in modal frequencies as experiments proceed. Similarly, for the non-damaged example, Bridge 2, no such pattern can be observed in modal frequencies due to the intactness of the structure; still, mild outliers occur in some cases. Instead, the modal frequencies from different datasets provide similar values regardless of the identification method. In contrast with the modal frequency parameters, it is challenging to associate the damping ratio with structural damage based on the existing datasets from Bridge 1. Likewise, in contrast with constant frequency values from different tests in Bridge 2, identified damping ratios change significantly, although no damage is introduced. Following system identification and modal analysis using various methods, the results merge into single and more reliable values through a method-centric ranking scheme. This decreases the impact of identification errors and enables removal of extreme values or outliers. It needs to be highlighted that the ranking approach's performance relies on its individual methods and is not necessarily bias-free despite removal of outliers.

The results show that further improvements are needed to capture damping behaviour better under low-amplitude and high-noise vibration testing characters. However, frequency is an indicative parameter as damage progresses, or relatively constant and stable if no damage is introduced. It should be noted that non-damaging environmental variants can also influence such behaviour, especially under long-term monitoring scenarios. Nevertheless, the synthesis of different identification methods provides a practical and technologically viable solution to improve the robustness of modal analysis and quantification of structural damage in real-life or large-scale infrastructure scenarios. The ranking approach is likely to improve identification accuracy in real-time operational modal analysis despite short time segments, especially if used with advanced automation techniques. Moreover, the proposed collaborative scheme is expected to be useful against novel bridge monitoring challenges such as citizen-engaged SHM and crowdsourcing-based modal identification, which will be addressed in future work.

\section{Acknowledgements}

The second author would like to acknowledge the funding provided by the Horizon 2020 Project, TURNkey, Grant No 821046. The shaking table tests are conducted at the University of Nevada, Reno, as an NSF-NEES project led by Prof. M.S. Saiidi and D. Sanders. System identification algorithms are 
made available by Prof. R. Betti from Columbia University, Prof. P. Van Overschee, and Prof. B. De Moor from KU Leuven. The authors are thankful for their contributions to this research.

\section{$\underline{\text { References }}$}

Abdelghani, M. V. (1998). Comparison study of subspace identification methods applied to flexible structures. Mechanical systems and signal processing, 12(5), 679-692.

$\mathrm{Au}, \mathrm{S}$. K. (2013). Bayesian operational modal analysis: theory, computation, practice. Computers \& Structures, 126, 3-14.

Banerjee, S. \&. (2008). Experimental verification of bridge seismic damage states quantified by calibrating analytical models with empirical field data. Earthquake Engineering and Engineering Vibration, 7(4), 383-393.

Banerjee, S., \& Chi, C. (2013). State-dependent fragility curves of bridges based on vibration measurements. Probabilistic Engineering Mechanics, 33, 116-125.

Brincker, R. V. (2001). Damping estimation by frequency domain decomposition. 19th International Modal Analysis Conference, 698-703.

Brincker, R. Z. (2001). Modal identification of output-only systems using frequency domain decomposition. Smart materials and structures, 10(3), 441-445.

Carden, E. P. (2004). Vibration based condition monitoring: a review. Structural health monitoring, 3(4), 355-377.

Chen, Y. F. (2008). Large-scale shake table test verification of bridge condition assessment methods. Journal of structural engineering, 134(7), 1235-1245.

Chen, Y., Feng, M. Q., \& Soyoz, S. (2008). Large-scale shake table test verification of bridge condition assessment methods. Journal of structural engineering, 134(7), 1235-1245.

Dervilis, N. W. (2015). On robust regression analysis as a means of exploring environmental and operational conditions for SHM data. Journal of Sound and Vibration, 347, 279-296.

Doebling, S. W. (1996). Damage identification and health monitoring of structural and mechanical systems from changes in their vibration characteristics: a literature review.

Doebling, S. W. (1998). A summary review of vibration-based damage identification methods. Shock and vibration digest, 30(2), 91-105.

Doebling, S. W., Farrar, C. R., Prime, M. B., \& Shevitz, D. W. (1996). Damage identification and health monitoring of structural and mechanical systems from changes in their vibration characteristics: a literature review (No. LA--13070-MS). Los Alamos.

Ebrahimi, R. E.-R. (2013). Vibration modeling and modification of cutting platform in a harvest combine by means of operational modal analysis (OMA). Measurement, 46(10), 3959-3967.

Ewins, D. J. (2009). Modal testing: theory, practice and application. John Wiley \& Sons.

Figueiredo, E. P. (2011). Machine learning algorithms for damage detection under operational and environmental variability. Structural Health Monitoring, 10(6), 559-572.

Frizzarin, M. F. (2010). Damage detection based on damping analysis of ambient vibration data. Structural Control and Health Monitoring, 17(4), 368-385.

Frizzarin, M., Feng, M. Q., Franchetti, P., Soyoz, S., \& Modena, C. (2010). Damage detection based on damping analysis of ambient vibration data. Structural Control and Health Monitoring, 17(4), 368-385.

Gelman, A. C. (2013). Bayesian data analysis. CRC press.

Giraldo, D. F. (2009). Modal identification through ambient vibration: comparative study. Journal of engineering mechanics, 135(8), 759-770.

Hastie, T., Tibshirani, R., \& Friedman, J. (2009). The elements of statistical learning. New York: Springer. 
Jacobsen, N. J. (2006). Using enhanced frequency domain decomposition as a robust technique to harmonic excitation in operational modal analysis. International Conference on Noise and Vibration Engineering.

Jafarkhani, R. \&. (2011). Finite element model updating using evolutionary strategy for damage detection. Computer- Aided Civil and Infrastructure Engineering, 26(3), 207-224.

Johnson, N. (2006). Large-scale experimental and analytical seismic studies of a two-span reinforced concrete bridge system. (No. 32-10946 UMI).

Johnson, N., Ranf, R. T., Saiidi, M. S., Sanders, D., \& Eberhard, M. (2008). Seismic testing of a twospan reinforced concrete bridge. Journal of Bridge Engineering, 13(2), 173-182.

Johnson, N., Saiidi, M. S., \& Sanders, D. (2009). Nonlinear earthquake response modeling of a largescale two-span concrete bridge. Journal of Bridge Engineering, 14(6), 460-471.

Johnson, N., Saiidi, M. S., \& Sanders, D. (2009). System versus component response of a two-span reinforced concrete bridge system. Bulletin of Earthquake Engineering, 7(2), 503-517.

Juang, J. N. (1985). An eigensystem realization algorithm for modal parameter identification and model reduction. Journal of guidance, control, and dynamics, 8(5), 620-627.

Juang, J. N. (1988). An eigensystem realization algorithm using data correlations (ERA/DC) for modal parameter identification. Journal of Control Theory and Advanced Technology, 4(1), 5-14.

Juang, J. N. (1993). Identification of observer/Kalman filter Markov parameters. Theory and experiments. Journal of Guidance, Control, and Dynamics, 16(2), 320-329.

Juang, J. N. (1997). System realization using information matrix. Journal of Guidance, Control, and Dynamics, 20(3), 492-500.

Kim, J. \&. (2011). Comparison study of output-only subspace and frequency-domain methods for system identification of base excited civil engineering structures. In Civil Engineering Topics, 4, 305-312.

Luş, H. B. (1999). Identification of linear structural systems using earthquake- induced vibration data. Earthquake Engineering \& Structural Dynamics, 28(11), 1449-1467.

Magalhães, F. C. (2007). Challenges in the application of stochastic modal identification methods to a cable-stayed bridge. J. Bridge Eng, 12, 746-754.

Mellinger, P. D. (2016). Variance estimation of modal parameters from output-only and input/output subspace-based system identification. Journal of Sound and Vibration, 379, 1-27.

Mottershead, J. E. (1993). Model updating in structural dynamics: a survey. Journal of sound and vibration. 167(2), 347-375.

Ozer, E., \& Feng, M. Q. (2016). Synthesizing spatiotemporally sparse smartphone sensor data for bridge modal identification. Smart Materials and Structures, 25(8), 085007.

Ozer, E., \& Feng, M. Q. (2017). Biomechanically influenced mobile and participatory pedestrian data for bridge monitoring. International Journal of Distributed Sensor Networks, 13(4), 1550147717705240.

Ozer, E., \& Feng, M. Q. (2017). Direction-sensitive smart monitoring of structures using heterogeneous smartphone sensor data and coordinate system transformation. Smart Materials and Structures, 26(4), 045026.

Ozer, E., \& Feng, M. Q. (2019). Structural Reliability Estimation with Participatory Sensing and Mobile Cyber-Physical Structural Health Monitoring Systems. Applied Sciences, 9(14), 2840.

Ozer, E., \& Soyoz, S. (2015). Vibration-based damage detection and seismic performance assessment of bridges. Earthquake Spectra, 31(1), 137-157.

Ozer, E., Feng, M. Q., \& Feng, D. (2015). Citizen sensors for SHM: Towards a crowdsourcing platform. Sensor, 15(6), 14591-14614.

Ozer, E., Feng, M. Q., \& Soyoz, S. (2014). SHM- integrated bridge reliability estimation using multivariate stochastic processes. Earthquake Engineering \& Structural Dynamics, 44(4), 601 618.

Peeters B., V. C. (2003). Comparative study of modal analysis techniques for bridge dynamic characteristics. Mechanical Systems and Signal Processing, 17(5), 965-988. 
Peeters, B. \&. (2001). Stochastic system identification for operational modal analysis: a review. Journal of Dynamic Systems, Measurement, and Control, 123(4), 659-667.

Phan, M. Q. (1992). Identification of linear systems by an asymptotically stable observer. NASA Langley Research Center Report L-16940 Hampton, VA: Langley. .

Phan, M. Q., Horta, L. G., Juang, J. N., \& Longman, R. W. (1992). Identification of linear systems by an asymptotically stable observer. NASA Langley Research Center Report L-16940 Hampton, VA: Langley.

Polikar, R. (2009). Ensemble learning. Scholarpedia, p. 4(1):2776.

Rahman, M. S. (2012). Comparison of system identification techniques with field vibration data for structural health monitoring of bridges, Master's Thesis. Carleton University.

Reynders, E. (2012). System identification methods for (operational) modal analysis: review and comparison. Archives of Computational Methods in Engineering, 19(1), 51-124.

Reynders, E. M. (2016). Uncertainty quantification in operational modal analysis with stochastic subspace identification: validation and applications. Mechanical Systems and Signal Processing, 66, 13-30.

Rytter, A. (1993). Vibrational based inspection of civil engineering structures. (Doctoral dissertation, Aalborg University, Aalborg, Denmark).

Saiidi, M. S. (2013). Shake Table Studies and Analysis of a Two-Span RC Bridge Model Subjected to a Fault Rupture. Journal of Bridge Engineering, 19(8).

Saiidi, M. S., Vosooghi, A., Choi, H., \& Somerville, P. (2013). Shake Table Studies and Analysis of a Two-Span RC Bridge Model Subjected to a Fault Rupture. Journal of Bridge Engineering, 19(8).

Salawu, O. S. (1997). Detection of structural damage through changes in frequency: a review. Engineering structures, 19(9), 718-723.

Siringoringo, D. M. (2006). Observed dynamic performance of the Yokohama- Bay Bridge from system identification using seismic records. Structural Control and Health Monitoring, 13(1), 226-244.

Soyoz, S., \& Feng, M. Q. (2008). Instantaneous damage detection of bridge structures and experimental verification. Structural Control and Health Monitoring. 15(7), 958-973.

Soyoz, S., \& Feng, M. Q. (2009). Long- term monitoring and identification of bridge structural parameters. Computer-Aided Civil and Infrastructure Engineering, 24(2), 82-92.

Soyoz, S., Feng, M. Q., \& Shinozuka, M. (2009). Structural reliability estimation with vibration-based identified parameters. Journal of engineering mechanics, 136(1), 100-106.

Tran, T. T., \& Ozer, E. (2020). Automated and Model-Free Bridge Damage Indicators with Simultaneous Multiparameter Modal Anomaly Detection. Sensors, 20(17), 4752.

Ubertini, F. C. (2017). Environmental effects on natural frequencies of the San Pietro bell tower in Perugia, Italy, and their removal for structural performance assessment. Mechanical Systems and Signal Processing, 82, 307-322.

Ubertini, F. G. (2013). Automated modal identification in operational conditions and its application to bridges. Engineering Structures, 46, 264-278.

Van Overschee P., D. M. (1996). Subspace identification for linear systems: Theory - Implementation Applications. Springer Science \& Business Media.

Van Overschee, P. \&. (1994). N4SID: Subspace algorithms for the identification of combined deterministic-stochastic systems. Automatica, 30(1), 75-93. 